\title{
Catalytic Asymmetric Arylative Cyclization of Alkynals: Phosphine-Free Rhodium/Diene Complexes as Efficient Catalysts
}

\author{
Ryo Shintani, Kazuhiro Okamoto, Yusuke Otomaru, Kazuhito Ueyama, and Tamio \\ Hayashi* $^{*}$ \\ Department of Chemistry, Graduate School of Science, Kyoto University, \\ Sakyo, Kyoto 606-8502, Japan \\ Supporting Information
}

\section{General}

All air- and moisture-sensitive manipulations were carried out with standard Schlenk techniques under nitrogen or in a glove box under argon.

$\mathrm{Et}_{2} \mathrm{O}, \mathrm{THF}$, and 1,4-dioxane were distilled over benzophenone ketyl under nitrogen. Methanol was distilled over magnesium turnings under nitrogen. Dichloromethane was distilled over $\mathrm{CaH}$ under nitrogen. DMF was distilled over $\mathrm{CaH}$ under vacuum.

Dimethyl malonate (Wako Chemicals), bromoacetaldehyde dimethyl acetal (Aldrich), bromopropionaldehyde dimethyl acetal (Wako Chemicals), 1-bromo-2butyne (Wako Chemicals), 1-bromo-2-pentyne (Wako Chemicals), benzyl bromide (Wako Chemicals), bromoacetone (TCI), p-toluenesulfonyl chloride (Wako Chemicals), iodomethane (Wako Chemicals), trimethylsilylacetylene (Aldrich), 3aminopropionaldehyde diethyl acetal (TCI), trimethyl orthoformate (Wako Chemicals), 4-dimethylaminopyridine (Wako Chemicals), $\mathrm{Et}_{3} \mathrm{~N}$ (Wako Chemicals), trifluoroacetic acid (Wako Chemicals), $p$-toluenesulfonic acid (Nacalai Tesque; monohydrate), $\mathrm{PhB}(\mathrm{OH})_{2}$ (TCI), NaOMe (Wako Chemicals), $\mathrm{K}_{2} \mathrm{CO}_{3}$ (Wako Chemicals), $\mathrm{NaH}$ (Kanto Chemicals; $60 \mathrm{wt} \%$ in mineral oil), $\mathrm{LiAlH}_{4}$ (Wako Chemicals), $n$-BuLi (Kanto Chemicals; $1.50 \mathrm{M}$ solution in hexane), CuI (Nacalai Tesque), $\mathrm{P}(t-\mathrm{Bu})_{3}$ (Kanto Chemicals), 1,3bis(diphenylphosphino)propane (Kanto Chemicals), 1,4-bis(diphenylphosphino)butane (TCI), 1,1'-bis(diphenylphosphino)ferrocene (Fluka), and $\mathrm{PPh}_{3}$ (Wako Chemicals) were used as received. Diisopropylamine (Aldrich) was distilled over $\mathrm{KOH}$ under vacuum prior to use. Other arylboronic acids were synthesized from the corresponding aryl bromides with $\mathrm{B}(\mathrm{OMe})_{3}$ (Wako Chemicals). $\left.\mathrm{PdCl}_{2}(\mathrm{PhCN})_{2}{ }^{1}, \quad \mathrm{RhCl}\left(\mathrm{C}_{2} \mathrm{H}_{4}\right)_{2}\right]_{2},{ }^{2}$ $[\mathrm{RhCl}(\mathrm{cod})]_{2},{ }^{3}[\mathrm{Rh}(\mathrm{OH})(\mathrm{cod})]_{2},{ }^{4}(R, R)-3,{ }^{5}$ and $(S, S)-4^{6}$ were synthesized following the literature procedures.

All other chemicals and solvents were purchased from Aldrich, Wako Chemicals, TCI, or Kanto Chemicals and used as received.

\footnotetext{
${ }^{1}$ Kharasch, M. S.; Seyler, R. C.; Mayo, F. R. J. Am. Chem. Soc. 1938, 60, 882.

${ }^{2}$ Cramer, R. Inorg. Synth. 1974, 15, 16.

${ }^{3}$ Giordano, G.; Crabtree, R. H. Inorg. Synth. 1979, 19, 218.

${ }^{4}$ Uson, R.; Oro, L. A.; Cabeza, J. A. Inorg. Synth. 1985, 23, 126.

${ }^{5}$ Hayashi, T.; Ueyama, K.; Tokunaga, N.; Yoshida, K. J. Am. Chem. Soc. 2003, 125, 11508.

${ }^{6}$ Tokunaga, N.; Otomaru, Y.; Okamoto, K.; Ueyama, K.; Shintani, R.; Hayashi, T. J. Am. Chem. Soc. 2004, 126, 13584.
} 


\section{Synthesis of Substrates}

The yields have not been optimized.

\section{3,3-Bis(benzyloxymethyl)-5-heptynal (1a)}

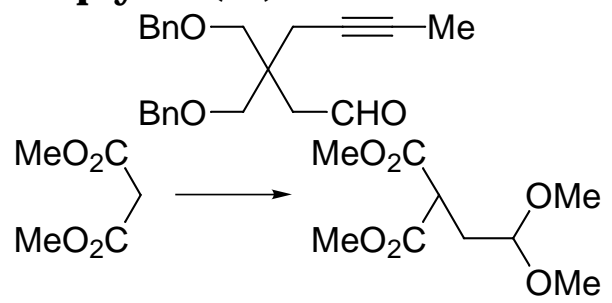

Dimethyl malonate $(2.45 \mathrm{~mL}, 21.3 \mathrm{mmol})$ was added to a solution of $\mathrm{NaOMe}(1.27 \mathrm{~g}$, $23.4 \mathrm{mmol})$ in $\mathrm{MeOH}(16 \mathrm{~mL})$, and the mixture was stirred for $15 \mathrm{~min}$ at $50{ }^{\circ} \mathrm{C}$. Bromoacetaldehyde dimethyl acetal $(3.00 \mathrm{~mL}, 22.5 \mathrm{mmol})$ was then added to this mixture, and the resulting solution was stirred for $72 \mathrm{~h}$ at $80^{\circ} \mathrm{C}$. After cooling to room temperature, the mixture was poured into $\mathrm{NaCl}$ (aqueous, saturated) and extracted with $\mathrm{Et}_{2} \mathrm{O}$. The organic layer was dried over $\mathrm{MgSO}_{4}$, filtered, and concentrated under vacuum. The residue was chromatographed on silica gel with EtOAc/hexane $=2 / 3$ to afford dimethyl 2,2-dimethoxyethylmalonate (CAS 51534-81-7) as a pale yellow oil (2.37 g, $10.8 \mathrm{mmol} ; 50 \%$ yield).

${ }^{1} \mathrm{H} \mathrm{NMR}\left(\mathrm{CDCl}_{3}\right): \delta 4.41\left(\mathrm{t},{ }^{3} J_{\mathrm{HH}}=5.5 \mathrm{~Hz}, 1 \mathrm{H}\right), 3.75(\mathrm{~s}, 6 \mathrm{H}), 3.54\left(\mathrm{t},{ }^{3} J_{\mathrm{HH}}=7.2 \mathrm{~Hz}, 1 \mathrm{H}\right)$, $3.32(\mathrm{~s}, 6 \mathrm{H}), 2.23\left(\mathrm{dd},{ }^{3} J_{\mathrm{HH}}=7.2\right.$ and $\left.5.5 \mathrm{~Hz}, 2 \mathrm{H}\right)$.

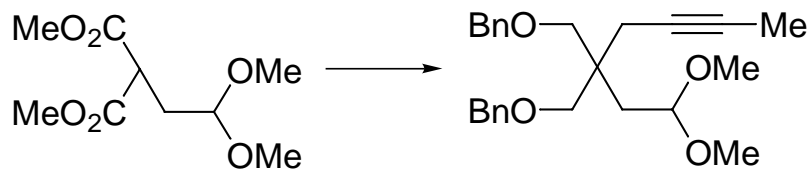

Dimethyl 2,2-dimethoxyethylmalonate $(2.18 \mathrm{~g}, 9.90 \mathrm{mmol})$ was added dropwisely to a suspension of $\mathrm{NaH}(478 \mathrm{mg}, 12.0 \mathrm{mmol} ; 60 \mathrm{wt} \%$ in mineral oil $)$ in THF $(50 \mathrm{~mL})$ at $0{ }^{\circ} \mathrm{C}$, and the mixture was stirred for $30 \mathrm{~min}$. 1-Bromo-2-butyne $(1.09 \mathrm{~mL}, 12.4 \mathrm{mmol})$ was then added to it at $0{ }^{\circ} \mathrm{C}$, and the resulting mixture was stirred for $4 \mathrm{~h}$ at room temperature. The reaction was quenched with water, and then extracted with $\mathrm{Et}_{2} \mathrm{O}$. The organic layer was dried over $\mathrm{MgSO}_{4}$, filtered, and concentrated under vacuum to afford 3,3-bis(methoxycarbonyl)-5-heptynal dimethyl acetal as a pale yellow oil quantitatively.

A solution of this oil in $\mathrm{Et}_{2} \mathrm{O}(15 \mathrm{~mL})$ was added to a suspension of $\mathrm{LiAlH}_{4}(758 \mathrm{mg}$, $20.0 \mathrm{mmol})$ in $\mathrm{Et}_{2} \mathrm{O}(25 \mathrm{~mL})$ at $0{ }^{\circ} \mathrm{C}$, and the mixture was stirred for $17 \mathrm{~h}$ at room temperature. After quenching with water, the precipitate was removed by filtration. The resulting solution was dried over $\mathrm{MgSO}_{4}$, filtered, and concentrated under vacuum to afford the diol as a colorless oil quantitatively.

A solution of this diol in THF $(10 \mathrm{~mL})$ was added to a suspension of $\mathrm{NaH}(1.22 \mathrm{~g}$, $30.5 \mathrm{mmol} ; 60 \mathrm{wt} \%$ in mineral oil) in THF $(30 \mathrm{~mL})$ and DMF $(5 \mathrm{~mL})$ at $0{ }^{\circ} \mathrm{C}$. After stirring for $15 \mathrm{~min}$ at $0{ }^{\circ} \mathrm{C}$, benzyl bromide $(2.59 \mathrm{~mL}, 21.8 \mathrm{mmol})$ was added to it, and the mixture was stirred for $36 \mathrm{~h}$ at room temperature. The reaction was quenched with water, and then extracted with $\mathrm{Et}_{2} \mathrm{O}$. The organic layer was dried over $\mathrm{MgSO}_{4}$, filtered, and concentrated under vacuum. The residue was chromatographed on silica gel with $\mathrm{Et}_{2} \mathrm{O} /$ hexane $=1 / 4$ to afford 3,3-bis(benzyloxymethyl)-5-heptynal dimethyl acetal as a colorless oil (2.26 g, $5.70 \mathrm{mmol} ; 58 \%$ yield).

${ }^{1} \mathrm{H}$ NMR $\left(\mathrm{CDCl}_{3}\right): \delta$ 7.35-7.29 $(\mathrm{m}, 8 \mathrm{H}), 7.29-7.23(\mathrm{~m}, 2 \mathrm{H}), 4.58\left(\mathrm{t},{ }^{3} J_{\mathrm{HH}}=5.5 \mathrm{~Hz}, 1 \mathrm{H}\right)$, 
$4.50(\mathrm{~s}, 4 \mathrm{H}), 3.43(\mathrm{~s}, 4 \mathrm{H}), 3.26(\mathrm{~s}, 6 \mathrm{H}), 2.31\left(\mathrm{q},{ }^{4} J_{\mathrm{HH}}=1.9 \mathrm{~Hz}, 2 \mathrm{H}\right), 1.77\left(\mathrm{~d},{ }^{3} J_{\mathrm{HH}}=5.5 \mathrm{~Hz}\right.$, $2 \mathrm{H}), 1.75\left(\mathrm{t},{ }^{4} J_{\mathrm{HH}}=2.4 \mathrm{~Hz}, 3 \mathrm{H}\right) .{ }^{13} \mathrm{C} \mathrm{NMR}\left(\mathrm{CDCl}_{3}\right): \delta 138.8,128.2,127.4,127.3,102.3,77.5$, 75.8, 73.2, 72.4, 52.5, 40.7, 34.7, 23.2, 3.6. Anal. Calcd for $\mathrm{C}_{25} \mathrm{H}_{32} \mathrm{O}_{4}: \mathrm{C}, 75.73 ; \mathrm{H}, 8.13$. Found: C, 75.52; $\mathrm{H}, 8.09$.

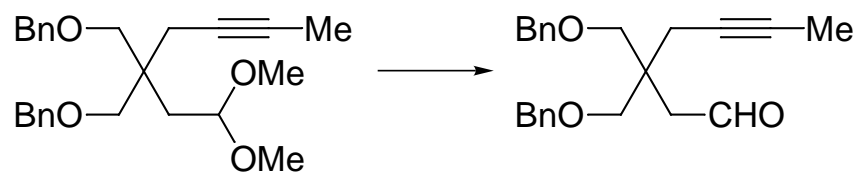

Trifluoroacetic acid (6 mL) was added to a mixture of 3,3-bis(benzyloxymethyl)-5heptynal dimethyl acetal $(1.93 \mathrm{~g}, 4.94 \mathrm{mmol})$ in $\mathrm{CHCl}_{3}(12 \mathrm{~mL})$ and water $(6 \mathrm{~mL})$ at $0{ }^{\circ} \mathrm{C}$. The resulting heterogeneous mixture was stirred for $9 \mathrm{~h}$ at room temperature. The reaction was quenched with $\mathrm{NaHCO}_{3}$ (aqueous, saturated), and then extracted with $\mathrm{CHCl}_{3}$. The organic layer was dried over $\mathrm{MgSO}_{4}$, filtered, and concentrated under vacuum. The residue was chromatographed on silica gel with $\mathrm{Et}_{2} \mathrm{O} /$ hexane $=1 / 2$ to afford 3,3-bis(benzyloxymethyl)-5-heptynal as a pale yellow oil $(1.17 \mathrm{~g}, 3.34 \mathrm{mmol} ; 68 \%$ yield).

${ }^{1} \mathrm{H}$ NMR $\left(\mathrm{CDCl}_{3}\right): \delta 9.80\left(\mathrm{t},{ }^{3} J_{\mathrm{HH}}=2.8 \mathrm{~Hz}, 1 \mathrm{H}\right), 7.35-7.31(\mathrm{~m}, 4 \mathrm{H}), 7.31-7.25(\mathrm{~m}, 6 \mathrm{H})$, $4.48(\mathrm{~s}, 4 \mathrm{H}), 3.52\left(\mathrm{~d},{ }^{2} J_{\mathrm{HH}}=8.9 \mathrm{~Hz}, 2 \mathrm{H}\right), 3.48\left(\mathrm{~d},{ }^{2} J_{\mathrm{HH}}=9.1 \mathrm{~Hz}, 2 \mathrm{H}\right), 2.49\left(\mathrm{~d},{ }^{3} J_{\mathrm{HH}}=2.8 \mathrm{~Hz}\right.$, $2 \mathrm{H}), 2.41\left(\mathrm{q},{ }^{4} \mathrm{~J}_{\mathrm{HH}}=2.6 \mathrm{~Hz}, 2 \mathrm{H}\right), 1.75\left(\mathrm{t},{ }^{4} J_{\mathrm{HH}}=2.5 \mathrm{~Hz}, 3 \mathrm{H}\right) .{ }^{13} \mathrm{C} \mathrm{NMR}\left(\mathrm{CDCl}_{3}\right): \delta 201.6$, 138.1, 128.2, 127.4, 127.3, 78.4, 74.8, 73.2, 72.3, 47.0, 42.6, 23.5, 3.4. HRMS (ESI) calcd for $\mathrm{C}_{23} \mathrm{H}_{26} \mathrm{O}_{3} \mathrm{Na}\left(\mathrm{M}+\mathrm{Na}^{+}\right)$373.1774, found 373.1775.

\section{3,3-Bis(benzyloxymethyl)-5-octynal (1b)}

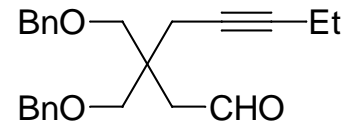

This was synthesized using 1-bromo-2-pentyne instead of 1-bromo-2-butyne, following the procedure for 3,3-bis(benzyloxymethyl)-5-heptynal. Pale yellow oil, 49\% overall yield from dimethyl 2,2-dimethoxyethylmalonate.

${ }^{1} \mathrm{H} \mathrm{NMR}\left(\mathrm{CDCl}_{3}\right): \delta 9.81\left(\mathrm{t},{ }^{3} J_{\mathrm{HH}}=2.8 \mathrm{~Hz}, 1 \mathrm{H}\right), 7.35-7.23(\mathrm{~m}, 10 \mathrm{H}), 4.48(\mathrm{~s}, 4 \mathrm{H}), 3.53(\mathrm{~d}$, $\left.{ }^{2} J_{\mathrm{HH}}=9.0 \mathrm{~Hz}, 2 \mathrm{H}\right), 3.49\left(\mathrm{~d},{ }^{2} J_{\mathrm{HH}}=9.0 \mathrm{~Hz}, 2 \mathrm{H}\right), 2.49\left(\mathrm{~d},{ }^{3} J_{\mathrm{HH}}=2.8 \mathrm{~Hz}, 2 \mathrm{H}\right), 2.42\left(\mathrm{t},{ }^{4} J_{\mathrm{HH}}=\right.$ $2.4 \mathrm{~Hz}, 2 \mathrm{H}), 2.12\left(\mathrm{qt}^{3} \mathrm{~J}_{\mathrm{HH}}=7.4 \mathrm{~Hz}\right.$ and $\left.{ }^{4} J_{\mathrm{HH}}=2.4 \mathrm{~Hz}, 2 \mathrm{H}\right), 1.08\left(\mathrm{t},{ }^{3} J_{\mathrm{HH}}=7.5 \mathrm{~Hz}, 3 \mathrm{H}\right) \cdot{ }^{13} \mathrm{C}$ NMR $\left(\mathrm{CDCl}_{3}\right): \delta 201.7,138.2,128.2,127.44,127.38,84.7,75.0,73.3,72.4,47.0,42.6,23.5$, 14.1, 12.3. HRMS (ESI) calcd for $\mathrm{C}_{24} \mathrm{H}_{28} \mathrm{O}_{3} \mathrm{Na}\left(\mathrm{M}+\mathrm{Na}^{+}\right)$387.1931, found 387.1938.

\section{4,4-Bis(benzyloxymethyl)-6-octyn-2-one (1f)

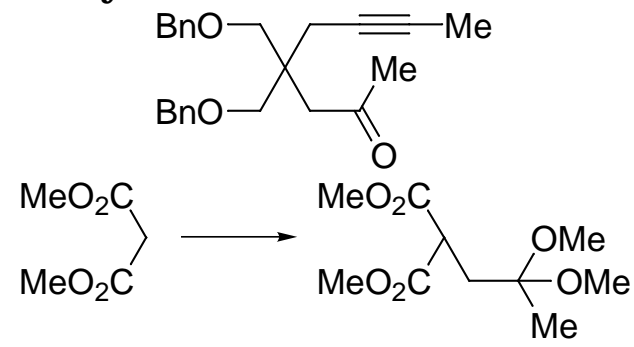

Dimethyl malonate $(2.29 \mathrm{~mL}, 20.0 \mathrm{mmol})$ was added to a suspension of $\mathrm{NaH}(880$ $\mathrm{mg}, 22.0 \mathrm{mmol} ; 60 \mathrm{wt} \%$ in mineral oil) in THF $(40 \mathrm{~mL})$ at $0{ }^{\circ} \mathrm{C}$, and the mixture was stirred for $15 \mathrm{~min}$ at $0{ }^{\circ} \mathrm{C}$. Bromoacetone $(2.24 \mathrm{~mL}, 24.0 \mathrm{mmol})$ was then added to it, and 
the resulting mixture was stirred for $15 \mathrm{~h}$ at room temperature. After being quenched with water, $\mathrm{NaCl}$ (aqueous, saturated) was added to it and the mixture was then extracted with $\mathrm{Et}_{2} \mathrm{O}$. The organic layer was dried over $\mathrm{MgSO}_{4}$, filtered, and concentrated under vacuum. The residue was chromatographed on silica gel with $\mathrm{Et}_{2} \mathrm{O} /$ hexane $=2 / 1$ to afford dimethyl acetonylmalonate (CAS 24889-15-4) as a pale yellow oil (3.06 g, $16.3 \mathrm{mmol} ; 81 \%$ yield).

A solution of dimethyl acetonylmalonate $(1.21 \mathrm{~g}, 6.42 \mathrm{mmol})$ and $p$-toluenesulfonic acid $(7.5 \mathrm{mg}, 39 \mu \mathrm{mol}$; monohydrate) in trimethyl orthoformate $(8 \mathrm{~mL})$ and $\mathrm{MeOH}(2$ $\mathrm{mL}$ ) was refluxed for $56 \mathrm{~h}$. After cooling to room temperature, the mixture was poured into $\mathrm{NaCl}$ (aqueous, saturated) and extracted with $\mathrm{Et}_{2} \mathrm{O}$. The organic layer was dried over $\mathrm{MgSO}_{4}$, filtered, and concentrated under vacuum. The residue was chromatographed on silica gel with $\mathrm{Et}_{2} \mathrm{O} /$ hexane=1/1 to afford dimethyl 2,2dimethoxypropylmalonate (CAS 133494-04-9) as an almost colorless oil (1.22 g, 5.22 mmol; 81\% yield).

${ }^{1} \mathrm{H} \mathrm{NMR}\left(\mathrm{CDCl}_{3}\right): \delta 3.74(\mathrm{~s}, 6 \mathrm{H}), 3.51\left(\mathrm{t},{ }^{3} \mathrm{~J}_{\mathrm{HH}}=6.7 \mathrm{~Hz}, 1 \mathrm{H}\right), 3.16(\mathrm{~s}, 6 \mathrm{H}), 2.31\left(\mathrm{~d},{ }^{3} \mathrm{~J}_{\mathrm{HH}}=\right.$ $6.6 \mathrm{~Hz}, 2 \mathrm{H}), 1.25(\mathrm{~s}, 3 \mathrm{H})$.

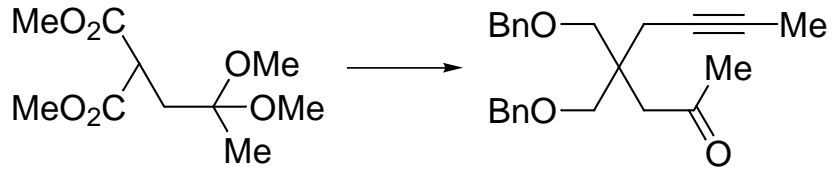

This was synthesized following the procedure for 3,3-bis(benzyloxymethyl)-5heptynal (1 a). Pale yellow oil, 50\% overall yield from dimethyl 2,2dimethoxypropylmalonate.

${ }^{1} \mathrm{H}$ NMR $\left(\mathrm{CDCl}_{3}\right): \delta$ 7.33-7.24 (m, 10H), $4.47(\mathrm{~s}, 4 \mathrm{H}), 3.52(\mathrm{~s}, 4 \mathrm{H}), 2.56(\mathrm{~s}, 2 \mathrm{H}), 2.42(\mathrm{q}$, $\left.{ }^{4} J_{\mathrm{HH}}=2.4 \mathrm{~Hz}, 2 \mathrm{H}\right), 2.10(\mathrm{~s}, 3 \mathrm{H}), 1.74\left(\mathrm{t},{ }^{4} J_{\mathrm{HH}}=2.5 \mathrm{~Hz}, 3 \mathrm{H}\right) .{ }^{13} \mathrm{C} \mathrm{NMR}\left(\mathrm{CDCl}_{3}\right): \delta 208.3$, $138.6,128.2,127.4,127.3,77.6,75.5,73.2,71.7,44.2,42.3,32.0,22.9,3.5$. Anal. Calcd for $\mathrm{C}_{24} \mathrm{H}_{28} \mathrm{O}_{3}$ : C, 79.09; H, 7.74. Found: C, 78.93; H, 7.76.

\section{(2-(1'-Propynyl)phenyl)acetaldehyde (1c)}

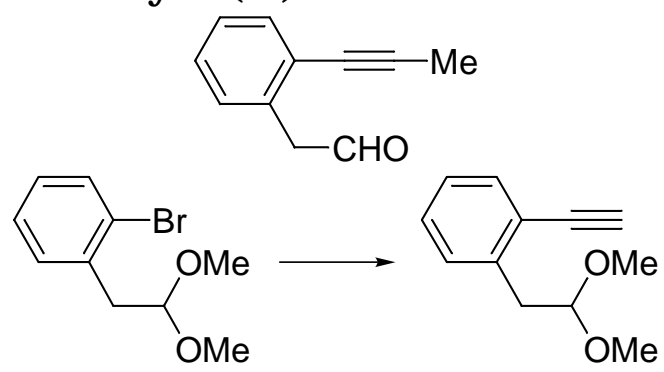

A solution of (2-bromophenyl)acetaldehyde dimethyl acetal ${ }^{7}$ (3.28 g, $13.4 \mathrm{mmol}^{\text {; CAS }}$ 112396-13-1) in 1,4-dioxane (15 mL) and trimethylsilylacetylene $(2.27 \mathrm{~mL}, 16.1 \mathrm{mmol})$ were successively added to a mixture of $\mathrm{PdCl}_{2}(\mathrm{PhCN})_{2}(154.2 \mathrm{mg}, 0.40 \mathrm{mmol}), \mathrm{P}(t-\mathrm{Bu})_{3}$ $(162.7 \mathrm{mg}, 0.80 \mathrm{mmol}), \mathrm{CuI}(51.0 \mathrm{mg}, 0.27 \mathrm{mmol})$, and $(i-\mathrm{Pr})_{2} \mathrm{NH}(2.25 \mathrm{~mL}, 16.1 \mathrm{mmol})$ in 1,4-dioxane $(15 \mathrm{~mL})$. The resulting mixture was stirred for $42 \mathrm{~h}$ at $50{ }^{\circ} \mathrm{C}$. After cooling to room temperature, this was passed through a pad of silica gel with $\mathrm{Et}_{2} \mathrm{O}$ and concentrated under vacuum. The residue was dissolved in $\mathrm{MeOH}(90 \mathrm{~mL})$, and $\mathrm{NaOH}$ $\left(30 \mathrm{~mL}\right.$; aqueous, $3 \mathrm{~N}$ ) was then added at $0{ }^{\circ} \mathrm{C}$. The reaction mixture was stirred for $1 \mathrm{~h}$

\footnotetext{
${ }^{7}$ Wünsch, B. Arch. Pharm. (Weinheim, Ger.) 1990, 323, 493-499.
} 
at room temperature, and then neutralized by $\mathrm{NH}_{4} \mathrm{Cl}$ (aqueous, saturated) at $0{ }^{\circ} \mathrm{C}$. After extraction with $\mathrm{Et}_{2} \mathrm{O}$, the organic layer was dried over $\mathrm{MgSO}_{4}$, flitered, and concentrated. The residue was chromatographed on silica gel with $\mathrm{Et}_{2} \mathrm{O} / \mathrm{hexane}=1 / 6$ to afford (2-ethynylphenyl)acetaldehyde dimethyl acetal as an orange oil (1.59 $\mathrm{g}, 8.36$ mmol; 63\% yield).

${ }^{1} \mathrm{H}$ NMR $\left(\mathrm{CDCl}_{3}\right): \delta 7.49\left(\mathrm{~d},{ }^{3} J_{\mathrm{HH}}=7.9 \mathrm{~Hz}, 1 \mathrm{H}\right), 7.30-7.27(\mathrm{~m}, 2 \mathrm{H}), 7.20-7.16(\mathrm{~m}, 1 \mathrm{H})$, $4.66\left(\mathrm{t},{ }^{3} \mathrm{~J}_{\mathrm{HH}}=5.6 \mathrm{~Hz}, 1 \mathrm{H}\right), 3.35(\mathrm{~s}, 6 \mathrm{H}), 3.30(\mathrm{~s}, 1 \mathrm{H}), 3.13\left(\mathrm{~d},{ }^{3} \mathrm{~J}_{\mathrm{HH}}=5.7 \mathrm{~Hz}, 2 \mathrm{H}\right) .{ }^{13} \mathrm{C} \mathrm{NMR}$ $\left(\mathrm{CDCl}_{3}\right): \delta 139.6,132.9,130.5,128.9,126.5,122.2,104.6,82.4,81.3,53.7,38.4$. Anal. Calcd for $\mathrm{C}_{12} \mathrm{H}_{14} \mathrm{O}_{2}$ : C, 75.76; $\mathrm{H}, 7.42$. Found: $\mathrm{C}, 75.70 ; \mathrm{H}, 7.55$.

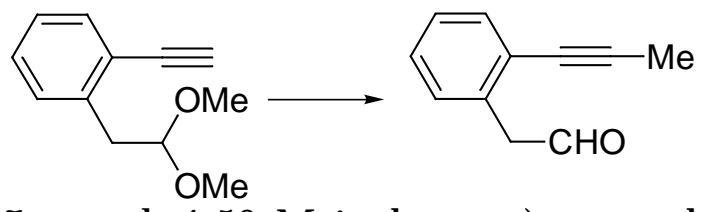

n-BuLi (5.58 mL, $8.37 \mathrm{mmol} ; 1.50 \mathrm{M}$ in hexane) was added to a solution of (2ethynylphenyl)acetaldehyde dimethyl acetal $(1.59 \mathrm{~g}, 8.37 \mathrm{mmol})$ in THF $(25 \mathrm{~mL})$ at -78 ${ }^{\circ} \mathrm{C}$. After stirring for $15 \mathrm{~min}$, iodomethane $(1.04 \mathrm{~mL}, 16.7 \mathrm{mmol})$ was added to it at -78 ${ }^{\circ} \mathrm{C}$. The reaction mixture was stirred for $3 \mathrm{~h}$ at room temperature and was quenched with water. After extraction with $\mathrm{Et}_{2} \mathrm{O}$, the organic layer was dried over $\mathrm{MgSO}_{4}$, flitered, and concentrated to afford (2-(1'-propynyl)phenyl)acetaldehyde dimethyl acetal as an orange oil $(1.63 \mathrm{~g})$. This orange oil $(736 \mathrm{mg})$ was dissolved in $\mathrm{CHCl}_{3}(10$ $\mathrm{mL})$ and water $(5 \mathrm{~mL})$ was then added. Trifluoroacetic acid $(5 \mathrm{~mL})$ was added to this at $0{ }^{\circ} \mathrm{C}$, and the resulting heterogeneous mixture was stirred for $21 \mathrm{~h}$ at room temperature. The reaction was quenched with $\mathrm{NaHCO}_{3}$ (aqueous, saturated), and then extracted with $\mathrm{CHCl}_{3}$. The organic layer was dried over $\mathrm{MgSO}_{4}$, filtered, and concentrated under vacuum. The residue was chromatographed on silica gel with $\mathrm{Et}_{2} \mathrm{O} /$ hexane $=1 / 3$ to afford (2-(1'-propynyl)phenyl)acetaldehyde as an orange-brown oil (395 mg, $2.50 \mathrm{mmol}$; $66 \%$ yield).

${ }^{1} \mathrm{H}$ NMR $\left(\mathrm{CDCl}_{3}\right): \delta 9.73\left(\mathrm{t},{ }^{3} \mathrm{~J}_{\mathrm{HH}}=2.2 \mathrm{~Hz}, 1 \mathrm{H}\right), 7.47-7.43(\mathrm{~m}, 1 \mathrm{H}), 7.30-7.22(\mathrm{~m}, 2 \mathrm{H})$, 7.21-7.17 (m, 1H), $3.83\left(\mathrm{~d},{ }^{3} \mathrm{~J}_{\mathrm{HH}}=2.0 \mathrm{~Hz}, 2 \mathrm{H}\right), 2.06(\mathrm{~s}, 3 \mathrm{H}) .{ }^{13} \mathrm{C} \mathrm{NMR}\left(\mathrm{CDCl}_{3}\right): \delta 199.7$, $134.2,132.3,130.0,128.1,127.4,124.6,91.1,78.0,49.2$, 4.3. Anal. Calcd for $\mathrm{C}_{11} \mathrm{H}_{10} \mathrm{O}: \mathrm{C}$, 83.51; $\mathrm{H}, 6.37$. Found: $\mathrm{C}, 83.25 ; \mathrm{H}, 6.51$.

\section{4,4-Bis(methoxycarbonyl)-6-octaynal (1d)

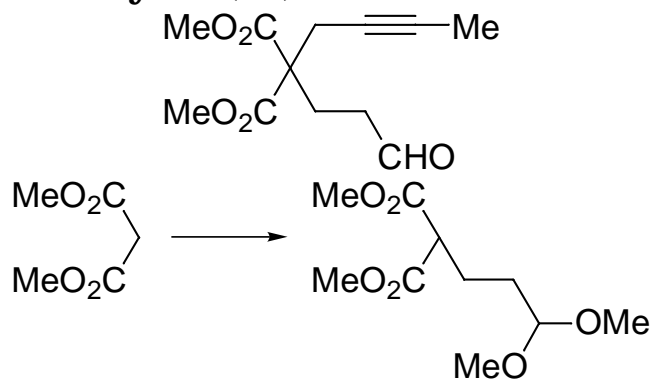

Dimethyl malonate $(2.29 \mathrm{~mL}, 20.0 \mathrm{mmol})$ was added to a solution of $\mathrm{NaOMe}(1.19 \mathrm{~g}$, $22.0 \mathrm{mmol})$ in $\mathrm{MeOH}(15 \mathrm{~mL})$, and the mixture was stirred for $15 \mathrm{~min}$ at $50{ }^{\circ} \mathrm{C}$. Bromopropionaldehyde dimethyl acetal $(3.28 \mathrm{~mL}, 24.0 \mathrm{mmol})$ was then added to this mixture, and the resulting solution was stirred for $61 \mathrm{~h}$ at $80^{\circ} \mathrm{C}$. After cooling to room temperature, the mixture was poured into $\mathrm{NaCl}$ (aqueous, saturated) and extracted 
with $\mathrm{Et}_{2} \mathrm{O}$. The organic layer was dried over $\mathrm{MgSO}_{4}$, filtered, and concentrated under vacuum. The residue was chromatographed on silica gel with $\mathrm{Et}_{2} \mathrm{O} /$ hexane $=1 / 2$ to afford dimethyl 3,3-dimethoxypropylmalonate (CAS 53744-52-8) as a pale yellow oil ( $2.72 \mathrm{~g}, 11.6 \mathrm{mmol} ; 58 \%$ yield).

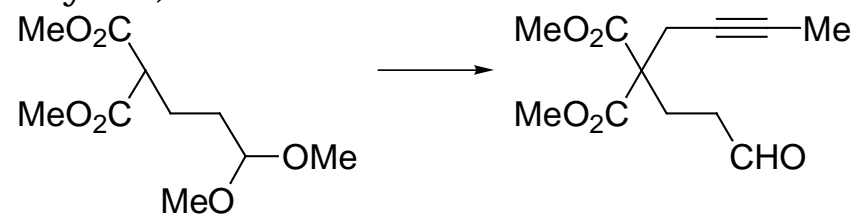

Dimethyl 3,3-dimethoxypropylmalonate $(1.43 \mathrm{~g}, 6.10 \mathrm{mmol})$ was added dropwisely to a suspension of $\mathrm{NaH}(292 \mathrm{mg}, 7.30 \mathrm{mmol} ; 60 \mathrm{wt} \%$ in mineral oil) in THF (23 mL) at room temperature, and the mixture was stirred for $20 \mathrm{~min}$. 1-Bromo-2-butyne (700 $\mu \mathrm{L}$; $8.00 \mathrm{mmol}$ ) was then added to it at $0{ }^{\circ} \mathrm{C}$, and the resulting mixture was stirred for $5 \mathrm{~h}$ at room temperature. The reaction was quenched with water, and then extracted with $\mathrm{Et}_{2} \mathrm{O}$. The organic layer was dried over $\mathrm{MgSO}_{4}$, filtered, and concentrated under vacuum to afford 4,4-bis(methoxycarbonyl)-6-octynal dimethyl acetal as a pale yellow oil quantitatively.

Trifluoroacetic acid $(8 \mathrm{~mL})$ was added to a mixture of 4,4-bis(methoxycarbonyl)-6octynal dimethyl acetal in $\mathrm{CHCl}_{3}(16 \mathrm{~mL})$ and water $(8 \mathrm{~mL})$ at $0{ }^{\circ} \mathrm{C}$. The resulting heterogeneous mixture was stirred for $7 \mathrm{~h}$ at $0{ }^{\circ} \mathrm{C}$. The reaction was quenched with $\mathrm{NaHCO}_{3}$ (aqueous, saturated), and then extracted with $\mathrm{CHCl}_{3}$. The organic layer was dried over $\mathrm{MgSO}_{4}$, filtered, and concentrated under vacuum. The residue was chromatographed on silica gel with $\mathrm{Et}_{2} \mathrm{O} /$ hexane=2/3 to afford 4,4bis(methoxycarbonyl)-6-octynal as a pale yellow oil (1.03 $\mathrm{g}, 4.29 \mathrm{mmol} ; 70 \%$ yield).

${ }^{1} \mathrm{H} \mathrm{NMR}\left(\mathrm{CDCl}_{3}\right): \delta 9.76\left(\mathrm{t},{ }^{3} \mathrm{~J}_{\mathrm{HH}}=1.2 \mathrm{~Hz}, 1 \mathrm{H}\right), 3.74(\mathrm{~s}, 6 \mathrm{H}), 2.77\left(\mathrm{q},{ }^{4} \mathrm{~J}_{\mathrm{HH}}=2.6 \mathrm{~Hz}, 2 \mathrm{H}\right)$, 2.52-2.48 (m 2H), 2.38-2.35 (m, 2H), $1.75\left(\mathrm{t},{ }^{4} \mathrm{~J}_{\mathrm{HH}}=2.5 \mathrm{~Hz}, 3 \mathrm{H}\right) .{ }^{13} \mathrm{C} \mathrm{NMR}\left(\mathrm{CDCl}_{3}\right): \delta 200.5$, $170.3,79.1,72.6,56.0,52.5,38.7,24.6,23.7,3.1$. HRMS (ESI) calcd for $\mathrm{C}_{12} \mathrm{H}_{16} \mathrm{O}_{5} \mathrm{Na}$ $\left(\mathrm{M}+\mathrm{Na}^{+}\right)$263.0890, found 263.0890.

\section{$N$-(2'-Pentynyl)- $N$-(p-tosyl)-3-aminopropionaldehyde (1e)}

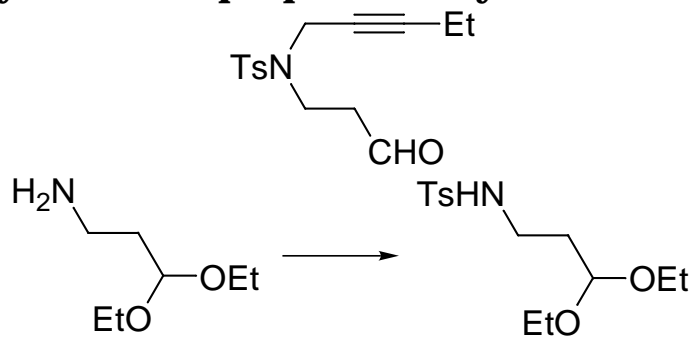

A solution of $p$-toluenesulfonyl chloride $(2.10 \mathrm{~g}, 11.0 \mathrm{mmol})$ in dichloromethane $(10$ $\mathrm{mL})$ was added to a mixture of 3-aminopropionaldehyde diethyl acetal $(1.62 \mathrm{~mL}, 10.0$ $\mathrm{mmol}), \mathrm{Et}_{3} \mathrm{~N}(2.79 \mathrm{~mL}, 20.0 \mathrm{mmol})$, and 4-dimethylaminopyridine $(6.1 \mathrm{mg}, 50 \mu \mathrm{mol})$ in dichloromethane $(20 \mathrm{~mL})$ at $0{ }^{\circ} \mathrm{C}$. The resulting mixture was stirred for $10 \mathrm{~h}$ at roomtemperature, and then quenched with water. After extraction with $\mathrm{Et}_{2} \mathrm{O}$, the organic layer was dried over $\mathrm{MgSO}_{4}$, filtered, and concentrated under vacuum to afford $N$-( $p$-tosyl)-3-aminopropionaldehyde diethyl acetal (CAS 164532-58-5) as a yellow oil qunatitatively.

${ }^{1} \mathrm{H} \mathrm{NMR}\left(\mathrm{CDCl}_{3}\right): \delta 7.74\left(\mathrm{~d},{ }^{3} J_{\mathrm{HH}}=8.0 \mathrm{~Hz}, 2 \mathrm{H}\right), 7.30\left(\mathrm{~d},{ }^{3} J_{\mathrm{HH}}=8.4 \mathrm{~Hz}, 2 \mathrm{H}\right), 5.11(\mathrm{bs}$, $1 \mathrm{H}), 4.48\left(\mathrm{t},{ }^{3} J_{\mathrm{HH}}=4.9 \mathrm{~Hz}, 1 \mathrm{H}\right), 3.61\left(\mathrm{dq},{ }^{2} J_{\mathrm{HH}}=8.8 \mathrm{~Hz}\right.$ and $\left.{ }^{3} J_{\mathrm{HH}}=7.2 \mathrm{~Hz}, 2 \mathrm{H}\right), 3.43(\mathrm{dq}$, 
${ }^{2} J_{\mathrm{HH}}=8.8 \mathrm{~Hz}$ and $\left.{ }^{3} J_{\mathrm{HH}}=7.1 \mathrm{~Hz}, 2 \mathrm{H}\right), 3.05\left(\mathrm{td},{ }^{3} J_{\mathrm{HH}}=6.1 \mathrm{~Hz}\right.$ and $\left.5.9 \mathrm{~Hz}, 2 \mathrm{H}\right), 2.42(\mathrm{~s}, 3 \mathrm{H})$, $1.76\left(\mathrm{dt},{ }^{3} J_{\mathrm{HH}}=6.2 \mathrm{~Hz}\right.$ and $\left.5.3 \mathrm{~Hz}, 2 \mathrm{H}\right), 1.17\left(\mathrm{t},{ }^{3} J_{\mathrm{HH}}=7.1 \mathrm{~Hz}, 6 \mathrm{H}\right)$.

TSHN

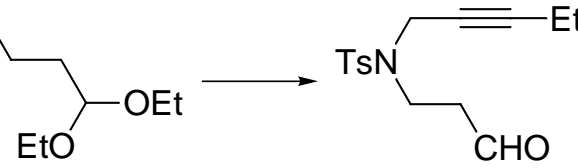

1-Bromo-2-pentyne $(530 \mu \mathrm{L}, 5.18 \mathrm{mmol})$ was added to a mixture of $\mathrm{N}$-( $p$-tosyl)-3propionacetaldehyde diethyl acetal $(1.12 \mathrm{~g}$, $3.72 \mathrm{mmol})$ and $\mathrm{K}_{2} \mathrm{CO}_{3}(1.02 \mathrm{~g}, 7.38 \mathrm{mmol})$ in DMF (12 mL) at room temperature, and the resulting mixture was stirred for $20 \mathrm{~h}$ at 80 ${ }^{\circ} \mathrm{C}$. The reaction was quenched with water, and then extracted with $\mathrm{Et}_{2} \mathrm{O}$. The organic layer was dried over $\mathrm{MgSO}_{4}$, filtered, and concentrated under vacuum. The residue was chromatographed on silica gel with $\mathrm{Et}_{2} \mathrm{O} /$ hexane $=1 / 2$ to afford $N-\left(2^{\prime}\right.$-pentynyl)- $N$ ( $p$-tosyl)-3-aminopropionaldehyde diethyl acetal as a pale yellow oil $(1.27 \mathrm{~g}, 3.46 \mathrm{mmol}$; $93 \%$ yield).

${ }^{1} \mathrm{H} \mathrm{NMR}\left(\mathrm{CDCl}_{3}\right): \delta 7.73\left(\mathrm{~d},{ }^{3} J_{\mathrm{HH}}=8.3 \mathrm{~Hz}, 2 \mathrm{H}\right), 7.28\left(\mathrm{~d},{ }^{3} J_{\mathrm{HH}}=8.1 \mathrm{~Hz}, 2 \mathrm{H}\right), 4.59\left(\mathrm{t},{ }^{3} J_{\mathrm{HH}}\right.$ $=5.5 \mathrm{~Hz}, 1 \mathrm{H}), 4.09\left(\mathrm{t},{ }^{4} J_{\mathrm{HH}}=2.2 \mathrm{~Hz}, 2 \mathrm{H}\right), 3.66\left(\mathrm{dq},{ }^{2} J_{\mathrm{HH}}=9.3 \mathrm{~Hz}\right.$ and $\left.{ }^{3} J_{\mathrm{HH}}=7.1 \mathrm{~Hz}, 2 \mathrm{H}\right)$, $3.52\left(\mathrm{dq},{ }^{2} J_{\mathrm{HH}}=9.2 \mathrm{~Hz}\right.$ and $\left.{ }^{3} J_{\mathrm{HH}}=7.0 \mathrm{~Hz}, 2 \mathrm{H}\right), 3.26\left(\mathrm{t},{ }^{3} J_{\mathrm{HH}}=7.0 \mathrm{~Hz}, 2 \mathrm{H}\right), 2.41(\mathrm{~s}, 3 \mathrm{H})$, $1.95-1.87(\mathrm{~m}, 4 \mathrm{H}), 1.21\left(\mathrm{t},{ }^{3} J_{\mathrm{HH}}=7.0 \mathrm{~Hz}, 6 \mathrm{H}\right), 0.90\left(\mathrm{t},{ }^{3} J_{\mathrm{HH}}=7.5 \mathrm{~Hz}, 3 \mathrm{H}\right)$.

Trifluoroacetic acid $(1.0 \mathrm{~mL})$ was added to a mixture of $\mathrm{N}-\left(2^{\prime}\right.$-pentynyl)- $\mathrm{N}-(\mathrm{p}$-tosyl)3-aminopropionaldehyde dimethyl acetal $(366 \mathrm{mg}, 1.00 \mathrm{mmol})$ in $\mathrm{CHCl}_{3}(2 \mathrm{~mL})$ and water $(1 \mathrm{~mL})$ at $0{ }^{\circ} \mathrm{C}$. The resulting heterogeneous mixture was stirred for $8 \mathrm{~h}$ at room temperature. The reaction was quenched with $\mathrm{NaHCO}_{3}$ (aqueous, saturated), and then extracted with $\mathrm{CHCl}_{3}$. The organic layer was dried over $\mathrm{MgSO}_{4}$, filtered, and concentrated under vacuum. The residue was chromatographed on silica gel with $\mathrm{Et}_{2} \mathrm{O} /$ hexane $=1 / 2$ to afford $N$-(2'-pentynyl)- $N$-( $p$-tosyl)-3-aminopropionaldehyde as a pale yellow oil ( $224 \mathrm{mg}, 0.76 \mathrm{mmol} ; 76 \%$ yield).

${ }^{1} \mathrm{H} \mathrm{NMR}\left(\mathrm{CDCl}_{3}\right): \delta 9.80\left(\mathrm{t},{ }^{3} J_{\mathrm{HH}}=1.2 \mathrm{~Hz}, 1 \mathrm{H}\right), 7.74\left(\mathrm{~d},{ }^{3} J_{\mathrm{HH}}=8.3 \mathrm{~Hz}, 2 \mathrm{H}\right), 7.30\left(\mathrm{~d},{ }^{3} J_{\mathrm{HH}}\right.$ $=7.9 \mathrm{~Hz}, 2 \mathrm{H}), 4.08\left(\mathrm{t},{ }^{4} J_{\mathrm{HH}}=2.2 \mathrm{~Hz}, 2 \mathrm{H}\right), 3.50\left(\mathrm{t},{ }^{3} J_{\mathrm{HH}}=6.9 \mathrm{~Hz}, 2 \mathrm{H}\right), 2.83\left(\mathrm{td},{ }^{3} J_{\mathrm{HH}}=6.9 \mathrm{~Hz}\right.$ and $1.2 \mathrm{~Hz}, 2 \mathrm{H}), 2.42(\mathrm{~s}, 3 \mathrm{H}), 1.94\left(\mathrm{qt},{ }^{3} J_{\mathrm{HH}}=7.4 \mathrm{~Hz}\right.$ and $\left.{ }^{4} J_{\mathrm{HH}}=2.3 \mathrm{~Hz}, 2 \mathrm{H}\right), 0.91\left(\mathrm{t},{ }^{3} J_{\mathrm{HH}}=\right.$ $7.6 \mathrm{~Hz}, 3 \mathrm{H}) .{ }^{13} \mathrm{C} \mathrm{NMR}\left(\mathrm{CDCl}_{3}\right): \delta 200.2,143.6,135.5,129.4,127.8,87.8,71.8,43.0,40.4$, 38.0, 21.5, 13.4, 12.1. HRMS (ESI) calcd for $\mathrm{C}_{15} \mathrm{H}_{20} \mathrm{NO}_{3} \mathrm{~S}\left(\mathrm{M}+\mathrm{H}^{+}\right)$294.1158, found 294.1158 .

\section{Dimethyl (2-pentynyl)(2-methylallyl)malonate (5)}

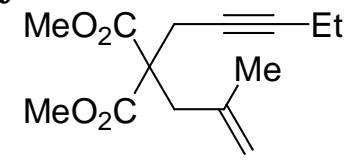

Dimethyl (2-methylallyl)malonate ${ }^{8}(215.6 \mathrm{mg}, 1.16 \mathrm{mmol})$ was added to a suspension of $\mathrm{NaH}\left(55.6 \mathrm{mg}, 1.39 \mathrm{mmol} ; 60 \mathrm{wt} \%\right.$ in mineral oil) in THF $(5 \mathrm{~mL})$ at $0{ }^{\circ} \mathrm{C}$, and the mixture was stirred for $15 \mathrm{~min}$. 1-Bromo-2-pentyne $(148 \mu \mathrm{L} ; 1.45 \mathrm{mmol})$ was then added to it at $0{ }^{\circ} \mathrm{C}$, and the resulting mixture was stirred for $3 \mathrm{~h}$ at room temperature. The reaction was quenched with water, and then extracted with $\mathrm{Et}_{2} \mathrm{O}$. The organic layer was dried over $\mathrm{MgSO}_{4}$, filtered, and concentrated under vacuum. The residue was

${ }^{8}$ Harrowven, D. C.; May, P. J.; Bradley, M. Tetrahedron Lett. 2003, 44, 503-506. 
chromatographed on silica gel with $\mathrm{Et}_{2} \mathrm{O} /$ hexane=1/4 to afford dimethyl (2pentynyl)(2-methylallyl)malonate as a pale yellow oil $(250.6 \mathrm{mg}, 0.99 \mathrm{mmol} ; 86 \%$ yield).

${ }^{1} \mathrm{H}$ NMR $\left(\mathrm{CDCl}_{3}\right): \delta 4.90(\mathrm{~s}, 1 \mathrm{H}), 4.82(\mathrm{~s}, 1 \mathrm{H}), 3.73(\mathrm{~s}, 6 \mathrm{H}), 2.83(\mathrm{~s}, 2 \mathrm{H}), 2.79\left(\mathrm{t},{ }^{4} J_{\mathrm{HH}}=\right.$ $2.4 \mathrm{~Hz}, 2 \mathrm{H}), 2.12\left(\mathrm{qt}^{3}{ }^{3} J_{\mathrm{HH}}=7.5 \mathrm{~Hz}\right.$ and $\left.{ }^{4} J_{\mathrm{HH}}=2.3 \mathrm{~Hz}, 2 \mathrm{H}\right), 1.66(\mathrm{~s}, 3 \mathrm{H}), 1.09\left(\mathrm{t},{ }^{3} J_{\mathrm{HH}}=7.4\right.$ $\mathrm{Hz}, 3 \mathrm{H}) .{ }^{13} \mathrm{C} \mathrm{NMR}\left(\mathrm{CDCl}_{3}\right): \delta 171.0,140.2,116.1,85.4,74.1,57.0,52.7,39.6,23.3,23.1$, 14.3, 12.4. Anal. Calcd for $\mathrm{C}_{14} \mathrm{H}_{20} \mathrm{O}_{4}: \mathrm{C}, 66.65 ; \mathrm{H}, 7.99$. Found: $\mathrm{C}, 66.41 ; \mathrm{H}, 8.18$. 


\section{Arylative Cyclization Reactions}

\section{General Procedure for Table 1.}

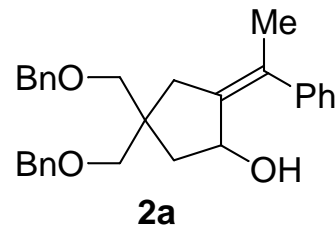

$\mathrm{KOH}\left(0.15 \mathrm{~mL}, 45 \mu \mathrm{mol} ; 0.3 \mathrm{M}\right.$ in $\left.\mathrm{H}_{2} \mathrm{O}\right)$ was added to a solution of $\left[\mathrm{RhCl}\left(\mathrm{C}_{2} \mathrm{H}_{4}\right)_{2}\right]_{2}$ $(2.0 \mathrm{mg}, 10 \mu \mathrm{mol} \mathrm{Rh})$ and ligand $(11.3 \mu \mathrm{mol})$ in 1,4-dioxane $(1.0 \mathrm{~mL})$, and the mixture was stirred for $5 \mathrm{~min}$ at room temperature. $\mathrm{PhB}(\mathrm{OH})_{2}(64.0 \mathrm{mg}, 0.52 \mathrm{mmol})$ and alkynal 1a $(52.6 \mathrm{mg}, 0.15 \mathrm{mmol})$ were added to it with additional 1,4-dioxane $(0.5 \mathrm{~mL})$, and the resulting solution was stirred for $4 \mathrm{~h}$ at $60^{\circ} \mathrm{C}$. The reaction mixture was directly passed through a pad of silica gel with $\mathrm{Et}_{2} \mathrm{O}$ and the solvent was removed under vacuum. The residue was purified by silica gel PTLC with $\mathrm{Et}_{2} \mathrm{O} /$ hexane $=1 / 2$ to afford the desired allylic alcohol $2 \mathbf{a}$ as a yellow oil.

${ }^{1} \mathrm{H}$ NMR $\left(\mathrm{CDCl}_{3}\right): \delta 7.37-7.27(\mathrm{~m}, 14 \mathrm{H}), 7.22-7.19(\mathrm{~m}, 1 \mathrm{H}), 4.62\left(\mathrm{~d},{ }^{2} J_{\mathrm{HH}}=12.1 \mathrm{~Hz}, 1 \mathrm{H}\right)$, $4.59\left(\mathrm{~d},{ }^{2} J_{\mathrm{HH}}=12.1 \mathrm{~Hz}, 1 \mathrm{H}\right), 4.49(\mathrm{~s}, 2 \mathrm{H}), 4.34\left(\mathrm{bt},{ }^{3} J_{\mathrm{HH}}=6.6 \mathrm{~Hz}, 1 \mathrm{H}\right), 3.59\left(\mathrm{~d},{ }^{2} J_{\mathrm{HH}}=8.5\right.$ $\mathrm{Hz}, 1 \mathrm{H}), 3.55\left(\mathrm{~d},{ }^{2} J_{\mathrm{HH}}=8.5 \mathrm{~Hz}, 1 \mathrm{H}\right), 3.43\left(\mathrm{~d},{ }^{3} J_{\mathrm{HH}}=7.7 \mathrm{~Hz}, 1 \mathrm{H}\right), 3.30\left(\mathrm{~d},{ }^{2} J_{\mathrm{HH}}=12.1 \mathrm{~Hz}\right.$, $1 \mathrm{H}), 3.28\left(\mathrm{~d},{ }^{2} J_{\mathrm{HH}}=12.0 \mathrm{~Hz}, 1 \mathrm{H}\right), 2.62\left(\mathrm{~d},{ }^{2} J_{\mathrm{HH}}=17.5 \mathrm{~Hz}, 1 \mathrm{H}\right), 2.42\left(\mathrm{~d},{ }^{2} J_{\mathrm{HH}}=17.3 \mathrm{~Hz}, 1 \mathrm{H}\right)$, $1.98(\mathrm{~s}, 3 \mathrm{H}), 1.82\left(\mathrm{dd},{ }^{2} J_{\mathrm{HH}}=14.4 \mathrm{~Hz}\right.$ and $\left.{ }^{2} J_{\mathrm{HH}}=6.0 \mathrm{~Hz}, 1 \mathrm{H}\right), 1.75\left(\mathrm{~d},{ }^{2} J_{\mathrm{HH}}=14.4 \mathrm{~Hz}, 1 \mathrm{H}\right)$. ${ }^{13} \mathrm{C}$ NMR $\left(\mathrm{CDCl}_{3}\right): \delta 143.2,141.5,138.4,137.8,131.4,128.4,128.3,128.0,127.83,127.81$, 127.7, 127.5, 127.4, 126.5, 76.0, 75.7, 73.6, 73.2, 72.3, 46.1, 42.9, 36.1, 21.4. Anal. Calcd for $\mathrm{C}_{29} \mathrm{H}_{32} \mathrm{O}_{3}: \mathrm{C}, 81.27 ; \mathrm{H}, 7.53$. Found: $\mathrm{C}, 81.07 ; \mathrm{H}, 7.64$.

\section{General Procedure for Table 2.}

A solution of $[\mathrm{Rh}(\mathrm{OH})(\mathrm{cod})]_{2}(2.4 \mathrm{mg}, 11 \mu \mathrm{mol} \mathrm{Rh}), \mathrm{PhB}(\mathrm{OH})_{2}(64.0 \mathrm{mg}, 0.52 \mathrm{mmol})$, and alkynal $(0.15 \mathrm{mmol})$ in 1,4-dioxane $(1.5 \mathrm{~mL})$ and water $(0.15 \mathrm{~mL})$ was stirred for $4 \mathrm{~h}$ at $60{ }^{\circ} \mathrm{C}$. The reaction mixture was directly passed through a pad of silica gel with $\mathrm{Et}_{2} \mathrm{O}$ and the solvent was removed under vacuum. The residue was purified by silica gel PTLC with $\mathrm{Et}_{2} \mathrm{O} /$ hexane to afford the desired allylic alcohol.

Pale yellow oil. $93 \%$ yield.

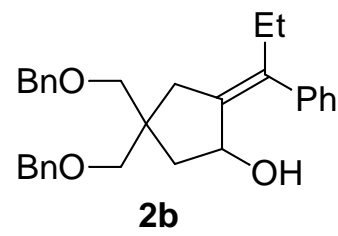

${ }^{1} \mathrm{H}$ NMR $\left(\mathrm{CDCl}_{3}\right): \delta 7.36-7.20(\mathrm{~m}, 15 \mathrm{H}), 4.60\left(\mathrm{~d},{ }^{2} J_{\mathrm{HH}}=12.2 \mathrm{~Hz}, 1 \mathrm{H}\right), 4.57\left(\mathrm{~d},{ }^{2} J_{\mathrm{HH}}=\right.$ $12.2 \mathrm{~Hz}, 1 \mathrm{H}), 4.50(\mathrm{~s}, 2 \mathrm{H}), 4.30\left(\mathrm{bt}^{3} J_{\mathrm{HH}}=6.1 \mathrm{~Hz}, 1 \mathrm{H}\right), 3.58\left(\mathrm{~d},{ }^{2} J_{\mathrm{HH}}=8.5 \mathrm{~Hz}, 1 \mathrm{H}\right), 3.55(\mathrm{~d}$, $\left.{ }^{2} J_{\mathrm{HH}}=8.5 \mathrm{~Hz}, 1 \mathrm{H}\right), 3.32\left(\mathrm{~d},{ }^{2} J_{\mathrm{HH}}=8.8 \mathrm{~Hz}, 1 \mathrm{H}\right), 3.29\left(\mathrm{~d},{ }^{2} J_{\mathrm{HH}}=8.8 \mathrm{~Hz}, 1 \mathrm{H}\right), 2.97\left(\mathrm{~d},{ }^{3} J_{\mathrm{HH}}=\right.$ $6.8 \mathrm{~Hz}, 1 \mathrm{H}), 2.60\left(\mathrm{~d},{ }^{2} J_{\mathrm{HH}}=17.0 \mathrm{~Hz}, 1 \mathrm{H}\right), 2.45\left(\mathrm{~d},{ }^{2} J_{\mathrm{HH}}=17.0 \mathrm{~Hz}, 1 \mathrm{H}\right), 2.37-2.30(\mathrm{~m}, 2 \mathrm{H})$, $1.81\left(\mathrm{dd},{ }^{2} J_{\mathrm{HH}}=14.3 \mathrm{~Hz}\right.$ and $\left.{ }^{3} J_{\mathrm{HH}}=6.2 \mathrm{~Hz}, 1 \mathrm{H}\right), 1.70\left(\mathrm{~d},{ }^{2} J_{\mathrm{HH}}=14.3 \mathrm{~Hz}, 1 \mathrm{H}\right), 0.90\left(\mathrm{t},{ }^{3} J_{\mathrm{HH}}=\right.$ $7.4 \mathrm{~Hz}, 3 \mathrm{H}) .{ }^{13} \mathrm{C} \mathrm{NMR}\left(\mathrm{CDCl}_{3}\right): \delta 141.8,140.7,138.5,138.2,138.0,128.40,128.37,128.3$, 
128.0, 127.7, 127.6, 127.5, 127.4, 126.5, 75.6, 75.4, 73.5, 73.2, 72.4, 46.1, 42.1, 35.1, 28.4, 12.4 . Anal. Calcd for $\mathrm{C}_{30} \mathrm{H}_{34} \mathrm{O}_{3}: \mathrm{C}, 81.41 ; \mathrm{H}, 7.74$. Found: C, 81.21; $\mathrm{H}, 7.81$.

Pale yellow solid. $76 \%$ yield.

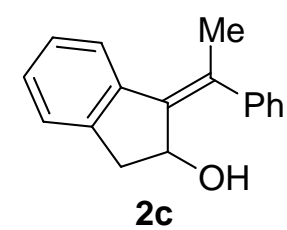

${ }^{1} \mathrm{H}$ NMR $\left(\mathrm{CDCl}_{3}\right): \delta 7.69\left(\mathrm{~d},{ }^{3} J_{\mathrm{HH}}=7.6 \mathrm{~Hz}, 1 \mathrm{H}\right), 7.42-7.37(\mathrm{~m}, 4 \mathrm{H}), 7.31-7.22(\mathrm{~m}, 4 \mathrm{H})$, $4.66\left(\mathrm{~d},{ }^{3} J_{\mathrm{HH}}=5.5 \mathrm{~Hz}, 1 \mathrm{H}\right), 3.13\left(\mathrm{dd},{ }^{2} J_{\mathrm{HH}}=16.6 \mathrm{~Hz}\right.$ and $\left.{ }^{3} J_{\mathrm{HH}}=5.7 \mathrm{~Hz}, 1 \mathrm{H}\right), 2.79\left(\mathrm{~d},{ }^{2} J_{\mathrm{HH}}=\right.$ $16.7 \mathrm{~Hz}, 1 \mathrm{H}), 2.43(\mathrm{~s}, 3 \mathrm{H}), 1.69$ (bs, $1 \mathrm{H}) .{ }^{13} \mathrm{C} \mathrm{NMR}\left(\mathrm{CDCl}_{3}\right): \delta 144.2,144.1,140.9,139.4$, 135.5, 128.4, 127.8, 127.7, 127.1, 126.6, 125.7, 125.6, 74.1, 40.6, 22.1. HRMS (ESI) calcd for $\mathrm{C}_{17} \mathrm{H}_{16} \mathrm{ONa}\left(\mathrm{M}+\mathrm{Na}^{+}\right) 259.1093$, found 259.1087.

Colorless oil. $75 \%$ yield.

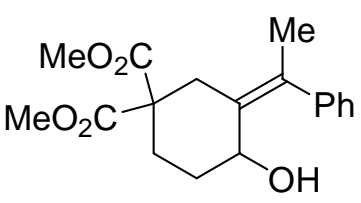

2d

${ }^{1} \mathrm{H}$ NMR $\left(\mathrm{CDCl}_{3}\right): \delta 7.32-7.29(\mathrm{~m}, 2 \mathrm{H}), 7.24\left(\mathrm{t},{ }^{3} \mathrm{~J}_{\mathrm{HH}}=7.3 \mathrm{~Hz}, 1 \mathrm{H}\right), 7.05\left(\mathrm{~d},{ }^{3} J_{\mathrm{HH}}=7.0\right.$ $\mathrm{Hz}, 2 \mathrm{H}), 4.30(\mathrm{bs}, 1 \mathrm{H}), 3.76(\mathrm{~s}, 3 \mathrm{H}), 3.75(\mathrm{~s}, 3 \mathrm{H}), 3.24\left(\mathrm{~d},{ }^{2} J_{\mathrm{HH}}=13.7 \mathrm{~Hz}, 1 \mathrm{H}\right), 2.87\left(\mathrm{~d},{ }^{2} J_{\mathrm{HH}}\right.$ $=13.8 \mathrm{~Hz}, 1 \mathrm{H}), 2.23-2.20(\mathrm{~m}, 2 \mathrm{H}), 2.01\left(\mathrm{~d},{ }^{4} J_{\mathrm{HH}}=1.2 \mathrm{~Hz}, 3 \mathrm{H}\right), 1.77-1.72(\mathrm{~m}, 2 \mathrm{H}), 1.21(\mathrm{bs}$, 1H). ${ }^{13} \mathrm{C}$ NMR $\left(\mathrm{CDCl}_{3}\right): \delta 172.3,171.0,143.4,135.3,131.0,128.3,127.6,126.6,66.5,56.5$, 52.7, 52.2, 30.4, 29.5, 25.5, 20.7. HRMS (ESI) calcd for $\mathrm{C}_{18} \mathrm{H}_{22} \mathrm{O}_{5} \mathrm{Na}\left(\mathrm{M}+\mathrm{Na}^{+}\right) 341.1359$, found 341.1357 .

Colorless viscous oil. $64 \%$ yield.

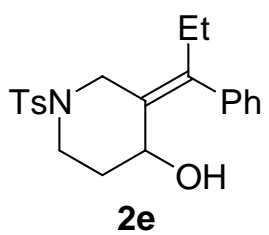

${ }^{1} \mathrm{H}$ NMR $\left(\mathrm{CDCl}_{3}\right): \delta 7.72\left(\mathrm{~d},{ }^{3} J_{\mathrm{HH}}=8.0 \mathrm{~Hz}, 2 \mathrm{H}\right), 7.36-7.24(\mathrm{~m}, 5 \mathrm{H}), 7.05\left(\mathrm{~d},{ }^{3} J_{\mathrm{HH}}=7.7\right.$ $\mathrm{Hz}, 2 \mathrm{H}), 4.57\left(\mathrm{~d},{ }^{2} J_{\mathrm{HH}}=12.7 \mathrm{~Hz}, 1 \mathrm{H}\right), 4.23-4.20(\mathrm{~m}, 1 \mathrm{H}), 3.65-3.61(\mathrm{~m}, 1 \mathrm{H}), 3.27\left(\mathrm{~d},{ }^{2} J_{\mathrm{HH}}=\right.$ $12.7 \mathrm{~Hz}, 1 \mathrm{H}), 2.85\left(\mathrm{td},{ }^{2} J_{\mathrm{HH}}=12.1 \mathrm{~Hz}\right.$ and $\left.{ }^{3} \mathrm{~J}_{\mathrm{HH}}=3.2 \mathrm{~Hz}, 1 \mathrm{H}\right), 2.57-2.44(\mathrm{~m}, 2 \mathrm{H}), 2.45(\mathrm{~s}$, $3 \mathrm{H}), 1.77\left(\mathrm{tdd},{ }^{2} J_{\mathrm{HH}}=14.2 \mathrm{~Hz}\right.$ and ${ }^{3} J_{\mathrm{HH}}=4.9 \mathrm{~Hz}$ and $\left.2.9 \mathrm{~Hz}, 1 \mathrm{H}\right), 1.70\left(\mathrm{dq},{ }^{2} J_{\mathrm{HH}}=13.8 \mathrm{~Hz}\right.$ and $\left.{ }^{3} J_{\mathrm{HH}}=2.7 \mathrm{~Hz}, 1 \mathrm{H}\right), 1.67(\mathrm{bs}, 1 \mathrm{H}), 0.94\left(\mathrm{t},{ }^{3} J_{\mathrm{HH}}=7.7 \mathrm{~Hz}, 3 \mathrm{H}\right) .{ }^{13} \mathrm{C}$ NMR $\left(\mathrm{CDCl}_{3}\right)$ : $\delta$ 143.4, 142.1, 141.0, 133.6, 129.6, 128.7, 128.2, 128.0, 127.7, 126.9, 64.9, 43.1, 40.9, 32.5, 27.4, 21.5, 12.8. HRMS (ESI) calcd for $\mathrm{C}_{21} \mathrm{H}_{25} \mathrm{NO}_{3} \mathrm{SNa}\left(\mathrm{M}+\mathrm{Na}^{+}\right) 394.1447$, found 394.1446.

General Procedure for Table 3.

$\mathrm{KOH}\left(0.15 \mathrm{~mL}, 45 \mu \mathrm{mol} ; 0.3 \mathrm{M}\right.$ in $\left.\mathrm{H}_{2} \mathrm{O}\right)$ was added to a solution of $\left[\mathrm{RhCl}\left(\mathrm{C}_{2} \mathrm{H}_{4}\right)_{2}\right]_{2}$ 
(2.0 $\mathrm{mg}, 10 \mu \mathrm{mol} \mathrm{Rh})$ and ligand $(11.3 \mu \mathrm{mol})$ in 1,4-dioxane $(1.0 \mathrm{~mL})$, and the mixture was stirred for $5 \mathrm{~min}$ at room temperature. $\mathrm{ArB}(\mathrm{OH})_{2}(0.53 \mathrm{mmol})$ and $1(0.15 \mathrm{mmol})$ were added to it with additional 1,4-dioxane $(0.5 \mathrm{~mL})$, and the resulting solution was stirred for $4 \mathrm{~h}$ at $60^{\circ} \mathrm{C}$. The reaction mixture was directly passed through a pad of silica gel with $\mathrm{Et}_{2} \mathrm{O}$ and the solvent was removed under vacuum. The residue was purified by silica gel PTLC with $\mathrm{Et}_{2} \mathrm{O} /$ hexane to afford the desired allylic alcohol.

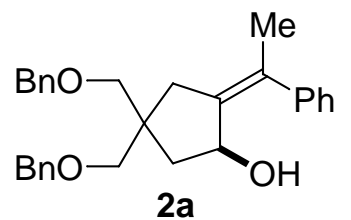

Entry 3. Yellow oil. 78\% yield. The ee was determined on a Daicel Chiralpak AD-H column with hexane $:$ isopropanol $=95: 5$, flow $=0.5 \mathrm{~mL} / \mathrm{min}$. Retention times: 22.8 $\min \left[(S)\right.$-enantiomer], 28.6 min $\left[(R)\right.$-enantiomer]. $95 \%$ ee. $[\alpha]^{20}{ }_{D}+51.0\left(c 1.02, \mathrm{CHCl}_{3}\right)$. The absolute configuration was determined by the Mosher's analysis after converting it to the corresponding $(R)$-MTPA ester. ${ }^{9}$

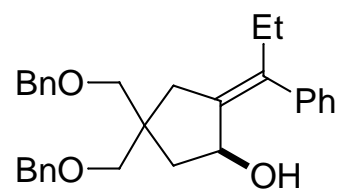

$2 b$

Entry 4. Pale yellow oil. $89 \%$ yield. The ee was determined on a Daicel Chiralpak AD$\mathrm{H}$ column with hexane : isopropanol $=95: 5$, flow $=0.5 \mathrm{~mL} / \mathrm{min}$. Retention times: 19.7 min $\left[(S)\right.$-enantiomer], 22.1 min $\left[(R)\right.$-enantiomer]. $94 \%$ ee. $[\alpha]^{20}{ }_{D}+48.9\left(c 0.80, \mathrm{CHCl}_{3}\right)$. The absolute configuration was assigned by analogy with entry 3 .

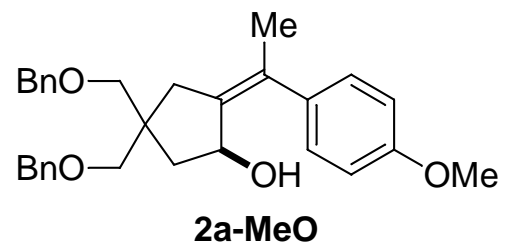

Entry 5. Yellow oil. 71\% yield. The ee was determined on a Daicel Chiralcel OD-H column with hexane : isopropanol $=90: 10$, flow $=0.8 \mathrm{~mL} / \mathrm{min}$. Retention times: 14.9 $\min \left[(R)\right.$-enantiomer], $20.3 \mathrm{~min}\left[(S)\right.$-enantiomer]. $93 \%$ ee. $[\alpha]^{20}{ }_{\mathrm{D}}+67.4\left(c 1.07, \mathrm{CHCl}_{3}\right)$. The absolute configuration was assigned by analogy with entry 3 .

${ }^{1} \mathrm{H} \mathrm{NMR}\left(\mathrm{CDCl}_{3}\right): \delta$ 7.37-7.26 (m, 12H), 6.89-6.82 $(\mathrm{m}, 2 \mathrm{H}), 4.62\left(\mathrm{~d},{ }^{2} J_{\mathrm{HH}}=12.1 \mathrm{~Hz}, 1 \mathrm{H}\right)$, $4.59\left(\mathrm{~d},{ }^{2} J_{\mathrm{HH}}=12.2 \mathrm{~Hz}, 1 \mathrm{H}\right), 4.49(\mathrm{~s}, 2 \mathrm{H}), 4.35\left(\mathrm{bt},{ }^{3} J_{\mathrm{HH}}=6.8 \mathrm{~Hz}, 1 \mathrm{H}\right), 3.79(\mathrm{~s}, 3 \mathrm{H}), 3.59(\mathrm{~d}$, $\left.{ }^{2} J_{\mathrm{HH}}=8.7 \mathrm{~Hz}, 1 \mathrm{H}\right), 3.55\left(\mathrm{~d},{ }^{2} J_{\mathrm{HH}}=8.6 \mathrm{~Hz}, 1 \mathrm{H}\right), 3.44\left(\mathrm{~d},{ }^{3} J_{\mathrm{HH}}=7.7 \mathrm{~Hz}, 1 \mathrm{H}\right), 3.30\left(\mathrm{~d},{ }^{2} J_{\mathrm{HH}}=\right.$ $12.1 \mathrm{~Hz}, 1 \mathrm{H}), 3.28\left(\mathrm{~d},{ }^{2} J_{\mathrm{HH}}=12.2 \mathrm{~Hz}, 1 \mathrm{H}\right), 2.60\left(\mathrm{~d},{ }^{2} J_{\mathrm{HH}}=17.4 \mathrm{~Hz}, 1 \mathrm{H}\right), 2.41\left(\mathrm{~d},{ }^{2} J_{\mathrm{HH}}=17.1\right.$ $\mathrm{Hz}, 1 \mathrm{H}), 1.96(\mathrm{~s}, 3 \mathrm{H}), 1.82\left(\mathrm{dd},{ }^{2} J_{\mathrm{HH}}=14.3 \mathrm{~Hz}\right.$ and $\left.{ }^{3} J_{\mathrm{HH}}=6.0 \mathrm{~Hz}, 1 \mathrm{H}\right), 1.75\left(\mathrm{dd},{ }^{2} J_{\mathrm{HH}}=14.4\right.$ $\mathrm{Hz}$ and $\left.{ }^{3} \mathrm{~J}_{\mathrm{HH}}=1.8 \mathrm{~Hz}, 1 \mathrm{H}\right) .{ }^{13} \mathrm{C} \mathrm{NMR}\left(\mathrm{CDCl}_{3}\right): \delta 158.1,140.9,138.5,137.8,135.6,130.9$,

9 (a) Dale, J. A.; Mosher, H. S. J. Am. Chem. Soc. 1973, 95, 512-519. (b) Ohtani, I.; Kusumi, T.; Kashman, Y.; Kakisawa, H. J. Am. Chem. Soc. 1991, 113, 4092-4096. 
$128.9,128.4,128.3,127.8,127.7,127.5,127.4,113.4,76.1,75.8,73.6,73.2,72.3,55.2,46.0$, 43.0, 36.3, 21.4. HRMS (ESI) calcd for $\mathrm{C}_{30} \mathrm{H}_{34} \mathrm{O}_{4} \mathrm{Na}\left(\mathrm{M}+\mathrm{Na}^{+}\right) 481.2349$, found 481.2344.

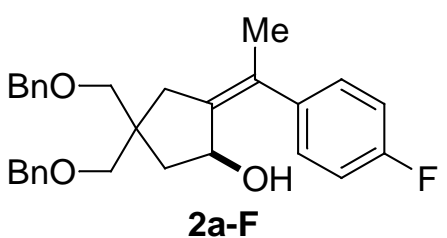

Entry 6. Yellow oil. 77\% yield. The ee was determined on a Daicel Chiralpak AD-H column with hexane $:$ isopropanol $=95: 5$, flow $=0.5 \mathrm{~mL} / \mathrm{min}$. Retention times: 23.4 min $\left[(S)\right.$-enantiomer], 29.3 min $\left[(R)\right.$-enantiomer]. $93 \%$ ee. $[\alpha]^{20}{ }_{D}+68.5\left(c 0.89, \mathrm{CHCl}_{3}\right)$. The absolute configuration was assigned by analogy with entry 3 .

${ }^{1} \mathrm{H}$ NMR $\left(\mathrm{CDCl}_{3}\right): \delta$ 7.37-7.27 $(\mathrm{m}, 12 \mathrm{H}), 7.01-6.96(\mathrm{~m}, 2 \mathrm{H}), 4.63\left(\mathrm{~d},{ }^{2} J_{\mathrm{HH}}=12.1 \mathrm{~Hz}, 1 \mathrm{H}\right)$, $4.59\left(\mathrm{~d},{ }^{2} J_{\mathrm{HH}}=12.1 \mathrm{~Hz}, 1 \mathrm{H}\right), 4.49(\mathrm{~s}, 2 \mathrm{H}), 4.26\left(\mathrm{bt},{ }^{3} J_{\mathrm{HH}}=6.8 \mathrm{~Hz}, 1 \mathrm{H}\right), 3.65\left(\mathrm{~d},{ }^{3} J_{\mathrm{HH}}=8.4\right.$ $\mathrm{Hz}, 1 \mathrm{H}), 3.59\left(\mathrm{~d},{ }^{2} J_{\mathrm{HH}}=8.7 \mathrm{~Hz}, 1 \mathrm{H}\right), 3.55\left(\mathrm{~d},{ }^{2} J_{\mathrm{HH}}=8.6 \mathrm{~Hz}, 1 \mathrm{H}\right), 3.28(\mathrm{~s}, 2 \mathrm{H}), 2.61\left(\mathrm{~d},{ }^{2} J_{\mathrm{HH}}\right.$ $=17.4 \mathrm{~Hz}, 1 \mathrm{H}), 2.41\left(\mathrm{~d},{ }^{2} J_{\mathrm{HH}}=17.2 \mathrm{~Hz}, 1 \mathrm{H}\right), 1.96(\mathrm{~s}, 3 \mathrm{H}), 1.82\left(\mathrm{dd},{ }^{2} J_{\mathrm{HH}}=14.4 \mathrm{~Hz}\right.$ and ${ }^{3} J_{\mathrm{HH}}$ $=5.7 \mathrm{~Hz}, 1 \mathrm{H}), 1.76\left(\mathrm{dd},{ }^{2} J_{\mathrm{HH}}=14.4 \mathrm{~Hz}\right.$ and $\left.{ }^{3} J_{\mathrm{HH}}=1.4 \mathrm{~Hz}, 1 \mathrm{H}\right) .{ }^{13} \mathrm{C} \mathrm{NMR}\left(\mathrm{CDCl}_{3}\right): \delta 161.5$ $\left(\mathrm{d},{ }^{1} J_{\mathrm{CF}}=245.0 \mathrm{~Hz}\right), 141.9,139.1\left(\mathrm{~d},{ }^{4} J_{\mathrm{CF}}=3.1 \mathrm{~Hz}\right), 138.4,137.7,130.3,129.5\left(\mathrm{~d},{ }^{3} J_{\mathrm{CF}}=7.8\right.$ $\mathrm{Hz}), 128.5,128.3,127.9,127.8,127.6,127.5,114.7\left(\mathrm{~d},{ }^{2} J_{\mathrm{CF}}=21.2 \mathrm{~Hz}\right), 76.3,76.0,73.7,73.3$, 72.3, 46.2, 43.3, 36.2, 21.4. HRMS (ESI) calcd for $\mathrm{C}_{29} \mathrm{H}_{31} \mathrm{FO}_{3} \mathrm{Na}\left(\mathrm{M}+\mathrm{Na}^{+}\right) 469.2149$, found 469.2147 .

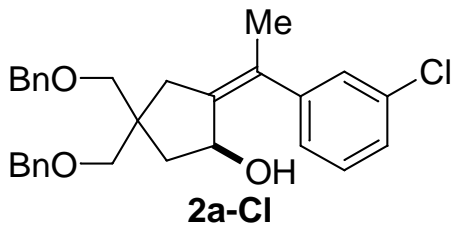

Entry 7. Yellow oil. 71\% yield. The ee was determined on a Daicel Chiralcel OD-H column with hexane : isopropanol $=95: 5$, flow $=0.5 \mathrm{~mL} / \mathrm{min}$. Retention times: 28.9 min $\left[(S)\right.$-enantiomer], 32.7 min $\left[(R)\right.$-enantiomer]. $96 \%$ ee. $[\alpha]^{20}{ }_{\mathrm{D}}+77.4\left(c \quad 0.86, \mathrm{CHCl}_{3}\right)$. The absolute configuration was assigned by analogy with entry 3 .

${ }^{1} \mathrm{H}$ NMR $\left(\mathrm{CDCl}_{3}\right): \delta 7.38-7.27(\mathrm{~m}, 14 \mathrm{H}), 7.24-7.17(\mathrm{~m}, 3 \mathrm{H}), 4.62\left(\mathrm{~d},{ }^{2} J_{\mathrm{HH}}=11.9 \mathrm{~Hz}, 1 \mathrm{H}\right)$, $4.59\left(\mathrm{~d},{ }^{2} J_{\mathrm{HH}}=12.0 \mathrm{~Hz}, 1 \mathrm{H}\right), 4.49(\mathrm{~s}, 2 \mathrm{H}), 4.28\left(\mathrm{bt},{ }^{3} J_{\mathrm{HH}}=6.6 \mathrm{~Hz}, 1 \mathrm{H}\right), 3.64\left(\mathrm{~d},{ }^{3} J_{\mathrm{HH}}=8.3\right.$ $\mathrm{Hz}, 1 \mathrm{H}), 3.58\left(\mathrm{~d},{ }^{2} J_{\mathrm{HH}}=8.1 \mathrm{~Hz}, 1 \mathrm{H}\right), 3.54\left(\mathrm{~d},{ }^{2} J_{\mathrm{HH}}=8.6 \mathrm{~Hz}, 1 \mathrm{H}\right), 3.27(\mathrm{~s}, 2 \mathrm{H}), 2.61\left(\mathrm{~d},{ }^{2} J_{\mathrm{HH}}\right.$ $=17.5 \mathrm{~Hz}, 1 \mathrm{H}), 2.41\left(\mathrm{~d},{ }^{2} J_{\mathrm{HH}}=17.5 \mathrm{~Hz}, 1 \mathrm{H}\right), 1.95(\mathrm{~s}, 3 \mathrm{H}), 1.82\left(\mathrm{dd},{ }^{2} J_{\mathrm{HH}}=14.4 \mathrm{~Hz}\right.$ and ${ }^{3} J_{\mathrm{HH}}$ $=5.7 \mathrm{~Hz}, 1 \mathrm{H}), 1.77\left(\mathrm{~d},{ }^{2} \mathrm{~J}_{\mathrm{HH}}=13.3 \mathrm{~Hz}, 1 \mathrm{H}\right) .{ }^{13} \mathrm{C} \mathrm{NMR}\left(\mathrm{CDCl}_{3}\right): \delta 145.0,142.8,138.4,137.7$, 133.8, 130.1, 129.2, 128.5, 128.3, 127.84, 127.83, 127.6, 127.5, 126.5, 126.3, 76.2, 75.8, 73.7, 73.3, 72.2, 46.2, 43.2, 36.3, 21.2. Anal. Calcd for $\mathrm{C}_{29} \mathrm{H}_{31} \mathrm{ClO}_{3}: \mathrm{C}, 75.23 ; \mathrm{H}, 6.75$. Found: $\mathrm{C}$, 74.95; $\mathrm{H}, 6.79$. 


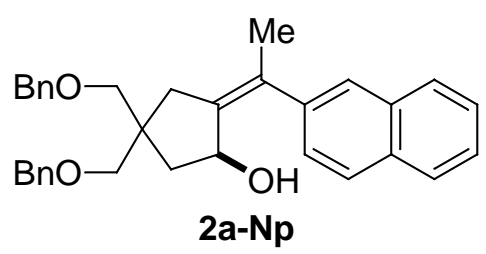

Entry 8. Yellow oil. 78\% yield. The ee was determined on a Daicel Chiralcel OD-H column with hexane : isopropanol $=90: 10$, flow $=0.5 \mathrm{~mL} / \mathrm{min}$. Retention times: 30.2 $\min \left[(R)\right.$-enantiomer], 41.5 min $\left[(S)\right.$-enantiomer]. $96 \%$ ee. $[\alpha]^{20}{ }_{\mathrm{D}}+42.1$ (c 1.18, $\left.\mathrm{CHCl}_{3}\right)$. The absolute configuration was assigned by analogy with entry 3 .

${ }^{1} \mathrm{H} \mathrm{NMR}\left(\mathrm{CDCl}_{3}\right): \delta$ 7.82-7.77 $(\mathrm{m}, 4 \mathrm{H}), 7.49-7.28(\mathrm{~m}, 13 \mathrm{H}), 4.63\left(\mathrm{~d},{ }^{2} J_{\mathrm{HH}}=12.3 \mathrm{~Hz}, 1 \mathrm{H}\right)$, $4.60\left(\mathrm{~d},{ }^{2} J_{\mathrm{HH}}=12.2 \mathrm{~Hz}, 1 \mathrm{H}\right), 4.51(\mathrm{~s}, 2 \mathrm{H}), 4.42\left(\mathrm{bt},{ }^{3} J_{\mathrm{HH}}=6.4 \mathrm{~Hz}, 1 \mathrm{H}\right), 3.60\left(\mathrm{~d},{ }^{2} J_{\mathrm{HH}}=8.6\right.$ $\mathrm{Hz}, 1 \mathrm{H}), 3.57\left(\mathrm{~d},{ }^{2} J_{\mathrm{HH}}=8.5 \mathrm{~Hz}, 1 \mathrm{H}\right), 3.45\left(\mathrm{~d},{ }^{3} J_{\mathrm{HH}}=7.4 \mathrm{~Hz}, 1 \mathrm{H}\right), 3.32\left(\mathrm{~d},{ }^{2} J_{\mathrm{HH}}=12.1 \mathrm{~Hz}\right.$, $1 \mathrm{H}), 3.31\left(\mathrm{~d},{ }^{2} J_{\mathrm{HH}}=12.0 \mathrm{~Hz}, 1 \mathrm{H}\right), 2.67\left(\mathrm{~d},{ }^{2} J_{\mathrm{HH}}=17.3 \mathrm{~Hz}, 1 \mathrm{H}\right), 2.47\left(\mathrm{~d},{ }^{2} J_{\mathrm{HH}}=17.5 \mathrm{~Hz}, 1 \mathrm{H}\right)$, $2.06(\mathrm{~s}, 3 \mathrm{H}), 1.84\left(\mathrm{dd},{ }^{2} J_{\mathrm{HH}}=14.3 \mathrm{~Hz}\right.$ and $\left.{ }^{3} J_{\mathrm{HH}}=5.8 \mathrm{~Hz}, 1 \mathrm{H}\right), 1.76\left(\mathrm{~d},{ }^{2} J_{\mathrm{HH}}=14.0 \mathrm{~Hz}, 1 \mathrm{H}\right)$. ${ }^{13} \mathrm{C}$ NMR $\left(\mathrm{CDCl}_{3}\right): \delta 142.2,140.7,138.5,137.9,133.3,132.2,131.4,128.5,128.4,128.1$, $127.9,127.8,127.57$ 127.55, 127.52, 127.51, 126.5, 126.4, 125.9, 125.6, 76.1, 75.8, 73.7, 73.3, 72.4, 46.2, 43.0, 36.3, 21.5. Anal. Calcd for $\mathrm{C}_{33} \mathrm{H}_{34} \mathrm{O}_{3}: \mathrm{C}, 82.81 ; \mathrm{H}, 7.16$. Found: $\mathrm{C}, 82.51 ; \mathrm{H}$, 7.29 .

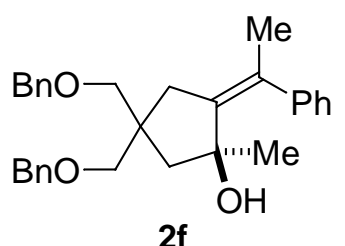

Entry 9. Colorless oil. $89 \%$ yield. The ee was determined on a Daicel Chiralpak AD-H column with hexane : isopropanol $=98: 2$, flow $=0.5 \mathrm{~mL} / \mathrm{min}$. Retention times: 18.4 min $\left[(S)\right.$-enantiomer], 28.3 min $\left[(R)\right.$-enantiomer]. $75 \%$ ee. $[\alpha]^{20}{ }_{D}+41.3\left(c 1.38, \mathrm{CHCl}_{3}\right)$. The absolute configuration was assigned by analogy with entry 3 .

${ }^{1} \mathrm{H}$ NMR $\left(\mathrm{CDCl}_{3}\right): \delta 7.35-7.25(\mathrm{~m}, 12 \mathrm{H}), 7.22-7.17(\mathrm{~m}, 3 \mathrm{H}), 4.61\left(\mathrm{~d},{ }^{2} J_{\mathrm{HH}}=12.0 \mathrm{~Hz}, 1 \mathrm{H}\right)$, $4.58\left(\mathrm{~d},{ }^{2} J_{\mathrm{HH}}=11.9 \mathrm{~Hz}, 1 \mathrm{H}\right), 4.51(\mathrm{~s}, 2 \mathrm{H}), 3.64\left(\mathrm{~d},{ }^{2} J_{\mathrm{HH}}=8.6 \mathrm{~Hz}, 1 \mathrm{H}\right), 3.59\left(\mathrm{~d},{ }^{2} J_{\mathrm{HH}}=8.7 \mathrm{~Hz}\right.$, $1 \mathrm{H}), 3.37\left(\mathrm{~d},{ }^{2} J_{\mathrm{HH}}=8.8 \mathrm{~Hz}, 1 \mathrm{H}\right), 3.33\left(\mathrm{~d},{ }^{2} J_{\mathrm{HH}}=8.8 \mathrm{~Hz}, 1 \mathrm{H}\right), 3.31(\mathrm{~s}, 1 \mathrm{H}), 2.70\left(\mathrm{~d},{ }^{2} J_{\mathrm{HH}}=\right.$ $17.2 \mathrm{~Hz}, 1 \mathrm{H}), 2.42\left(\mathrm{~d},{ }^{2} J_{\mathrm{HH}}=17.2 \mathrm{~Hz}, 1 \mathrm{H}\right), 1.91(\mathrm{~s}, 3 \mathrm{H}), 1.90\left(\mathrm{~d},{ }^{2} J_{\mathrm{HH}}=14.3 \mathrm{~Hz}, 1 \mathrm{H}\right), 1.81$ $\left(\mathrm{dd},{ }^{2} J_{\mathrm{HH}}=14.3 \mathrm{~Hz}, 1 \mathrm{H}\right), 0.89(\mathrm{~s}, 3 \mathrm{H}) .{ }^{13} \mathrm{C}$ NMR $\left(\mathrm{CDCl}_{3}\right): \delta 143.8,142.8,138.6,138.1,131.1$, 128.6, 128.4, 128.3, 127.8, 127.7, 127.6, 127.5, 127.4, 126.3, 78.1, 76.1, 75.6, 73.6, 73.3, 51.6, 44.1, 39.3, 28.4, 24.3. Anal. Calcd for $\mathrm{C}_{30} \mathrm{H}_{34} \mathrm{O}_{3}: \mathrm{C}, 81.41 ; \mathrm{H}, 7.74$. Found: $\mathrm{C}, 81.14 ; \mathrm{H}, 7.88$.

\section{Procedure for Equation 2.}

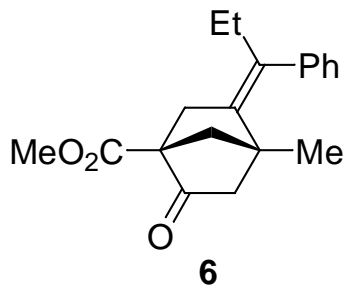

A solution of $[\mathrm{Rh}(\mathrm{OH})(\mathrm{cod})]_{2}(2.4 \mathrm{mg}, 11 \mu \mathrm{mol} \mathrm{Rh}), \mathrm{PhB}(\mathrm{OH})_{2}(64.0 \mathrm{mg}, 0.52 \mathrm{mmol})$, 
and compound $5(37.8 \mathrm{mg}, 0.15 \mathrm{mmol})$ in 1,4-dioxane $(1.5 \mathrm{~mL})$ and water $(0.15 \mathrm{~mL})$ was stirred for $5 \mathrm{~h}$ at $70{ }^{\circ} \mathrm{C}$. The reaction mixture was directly passed through a pad of silica gel with $\mathrm{Et}_{2} \mathrm{O}$ and the solvent was removed under vacuum. The residue was purified by silica gel PTLC with $\mathrm{Et}_{2} \mathrm{O} /$ hexane=1/3.5 to afford compound 6 as a white solid (39.5 $\mathrm{mg}, 0.13 \mathrm{mmol} ; 88 \%$ yield).

${ }^{1} \mathrm{H}$ NMR $\left(\mathrm{CDCl}_{3}\right): \delta 7.35-7.21(\mathrm{~m}, 3 \mathrm{H}), 7.17-6.92(\mathrm{~m}, 2 \mathrm{H}), 3.79(\mathrm{~s}, 3 \mathrm{H}), 2.95\left(\mathrm{~d},{ }^{2} J_{\mathrm{HH}}=\right.$ $16.3 \mathrm{~Hz}, 1 \mathrm{H}), 2.62\left(\mathrm{dd},{ }^{2} J_{\mathrm{HH}}=16.1 \mathrm{~Hz}\right.$ and $\left.{ }^{4} J_{\mathrm{HH}}=2.5 \mathrm{~Hz}, 1 \mathrm{H}\right), 2.31-2.24(\mathrm{~m}, 2 \mathrm{H}), 2.19-2.12$ $(\mathrm{m}, 2 \mathrm{H}), 2.03\left(\mathrm{dd},{ }^{2} J_{\mathrm{HH}}=10.5 \mathrm{~Hz}\right.$ and $\left.{ }^{4} J_{\mathrm{HH}}=4.0 \mathrm{~Hz}, 1 \mathrm{H}\right), 1.97\left(\mathrm{dd},{ }^{2} J_{\mathrm{HH}}=9.8 \mathrm{~Hz}\right.$ and ${ }^{4} J_{\mathrm{HH}}$ $=2.7 \mathrm{~Hz}, 1 \mathrm{H}), 0.86\left(\mathrm{t},{ }^{3} \mathrm{JHH}_{\mathrm{HH}}=7.1 \mathrm{~Hz}, 3 \mathrm{H}\right), 0.67(\mathrm{~s}, 3 \mathrm{H}) .{ }^{13} \mathrm{C} \mathrm{NMR}\left(\mathrm{CDCl}_{3}\right): \delta 210.5,170.5$, 141.8, 136.2, 135.5, 128.1, 127.8, 126.8, 63.2, 52.4, 52.1, 50.0, 47.8, 36.8, 29.9, 20.2, 11.9. Anal. Calcd for $\mathrm{C}_{19} \mathrm{H}_{22} \mathrm{O}_{3}$ : C, 76.48; $\mathrm{H}, 7.43$. Found: $\mathrm{C}, 76.11 ; \mathrm{H}, 7.60$. 


\section{IV. ${ }^{1} \mathrm{H}$ NMR Spectra}
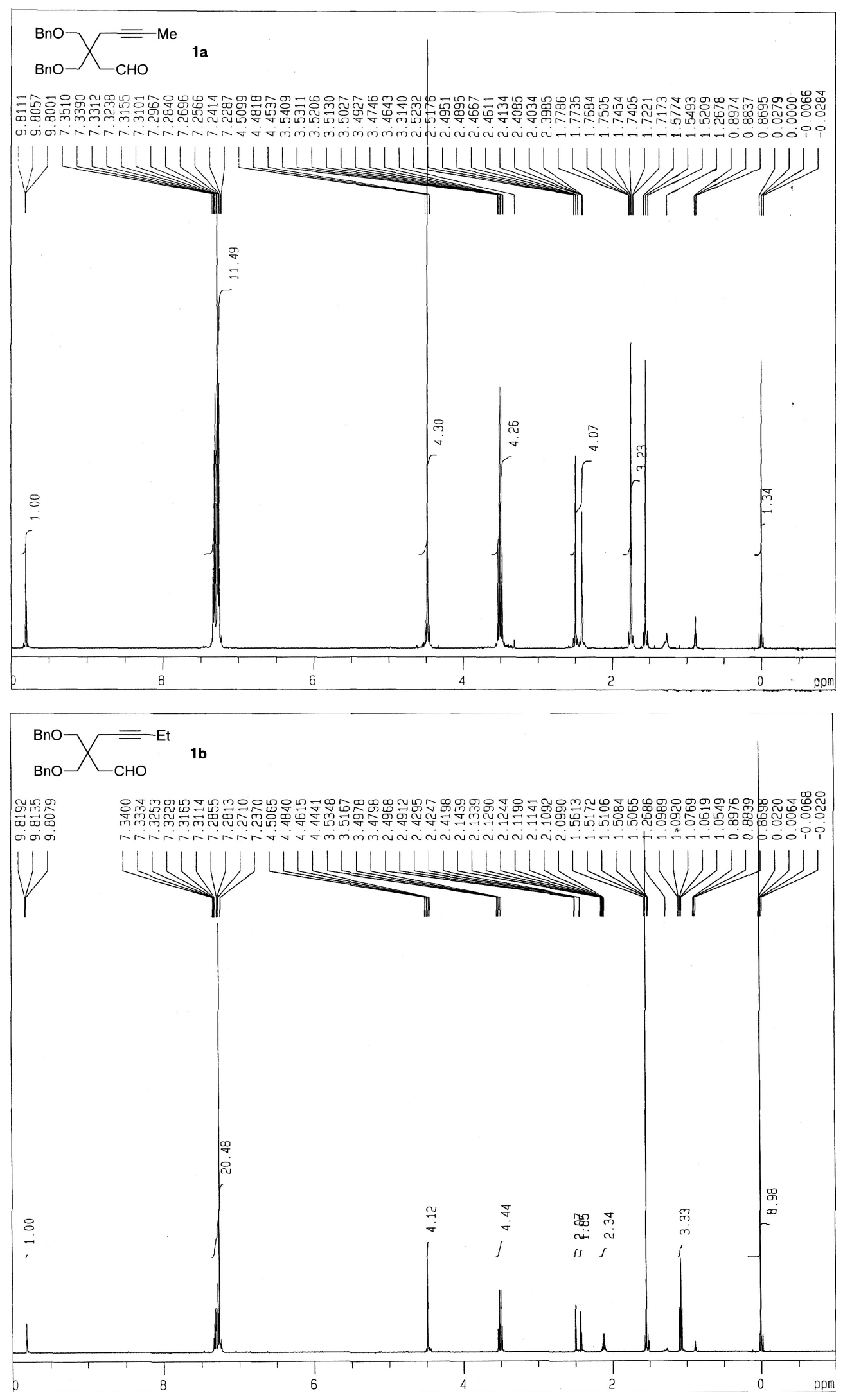

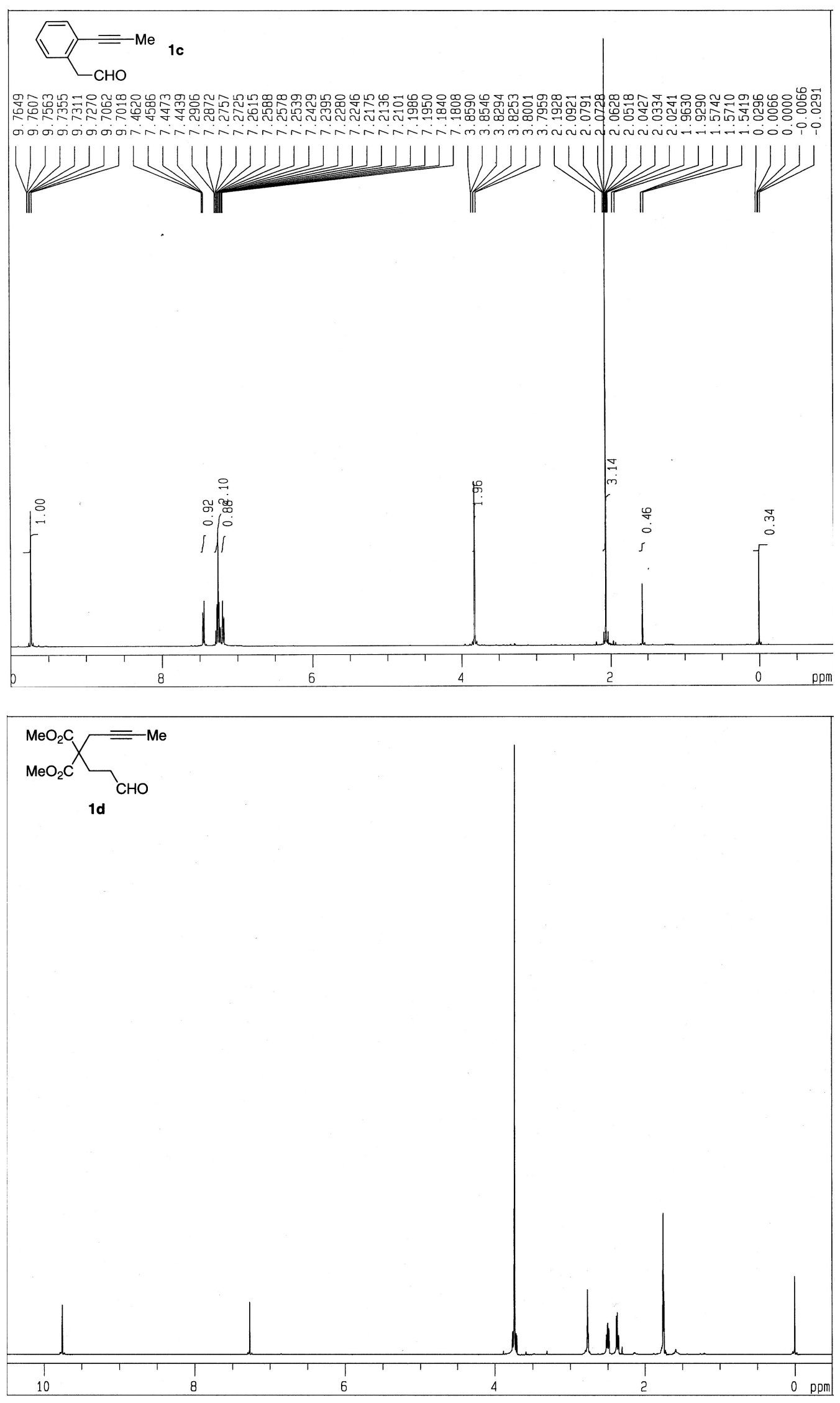

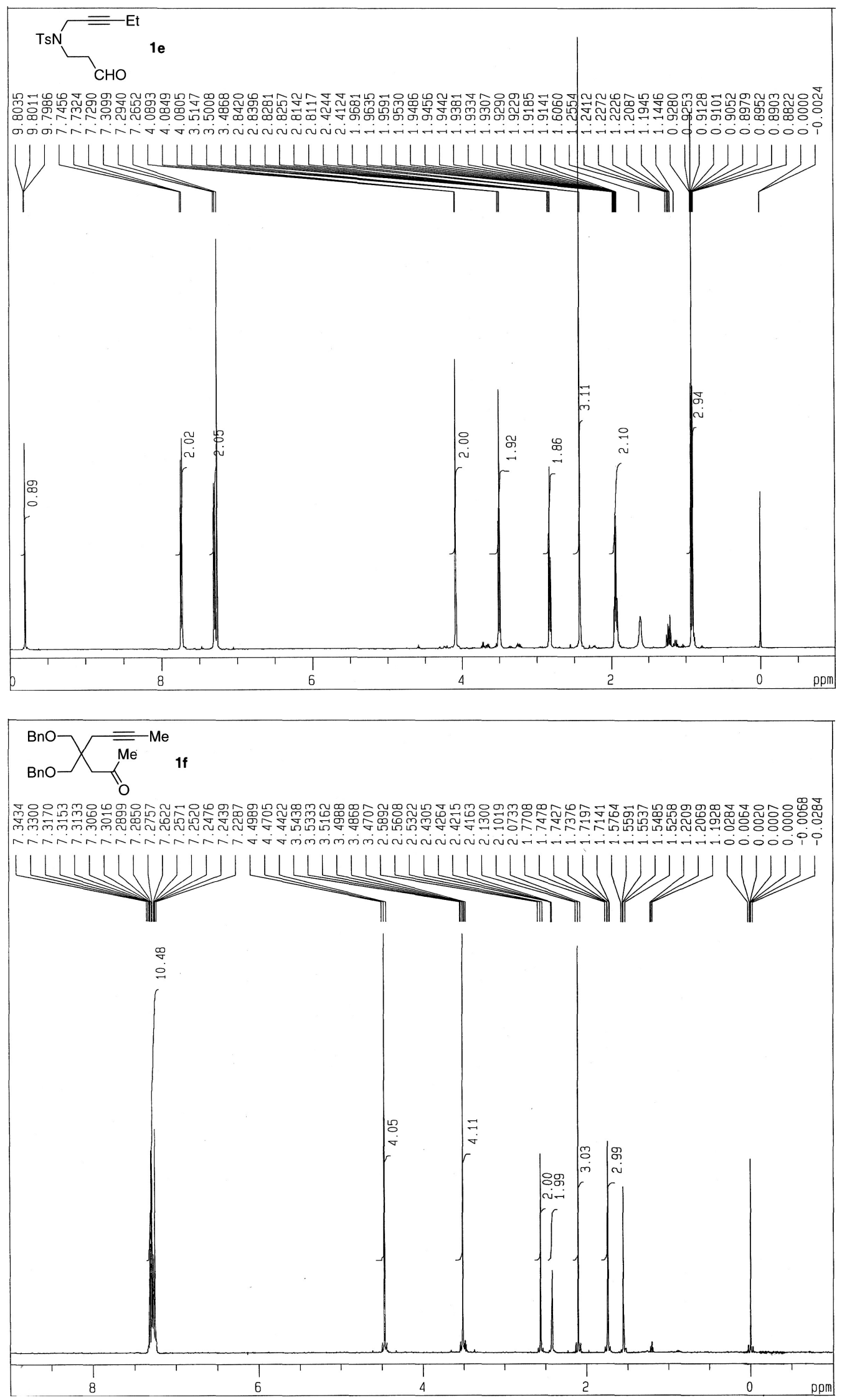

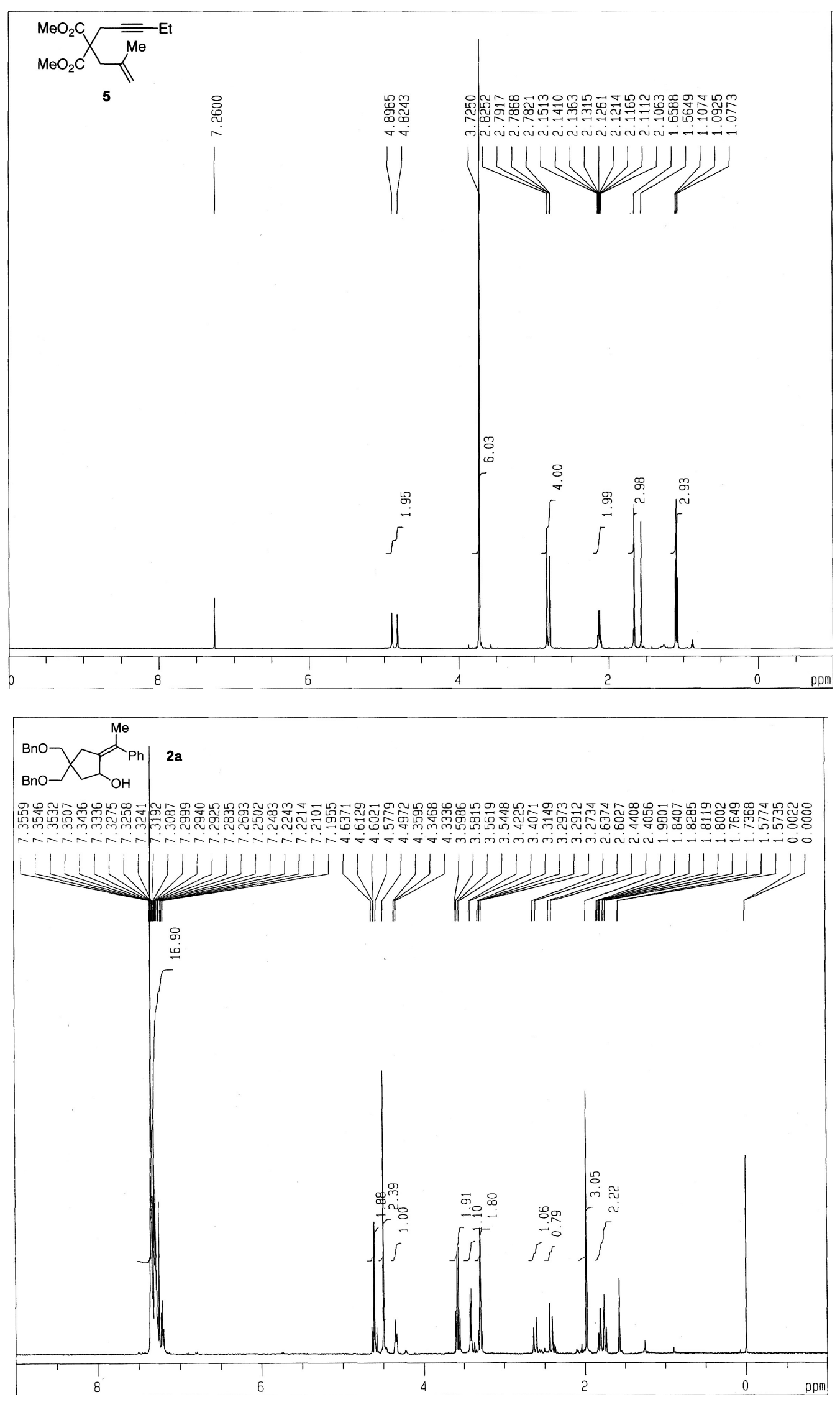

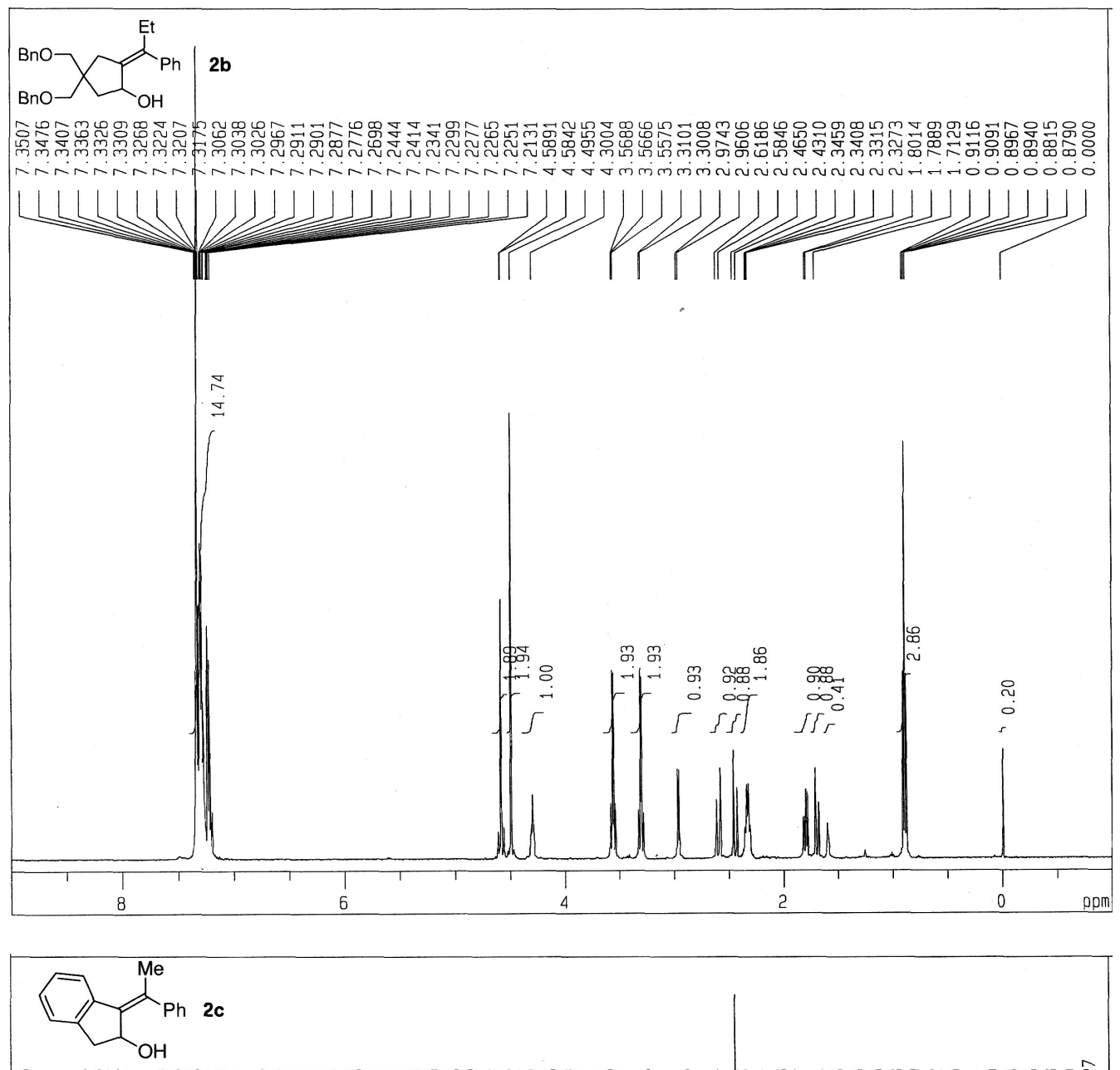

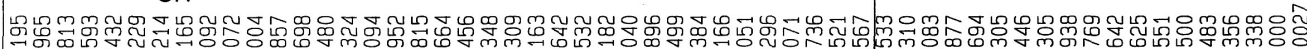

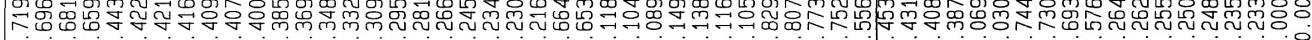

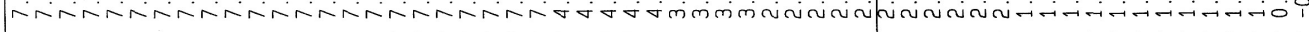

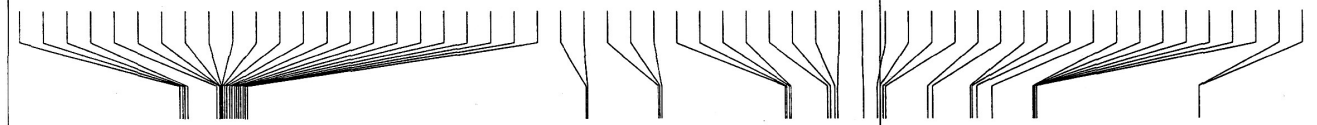

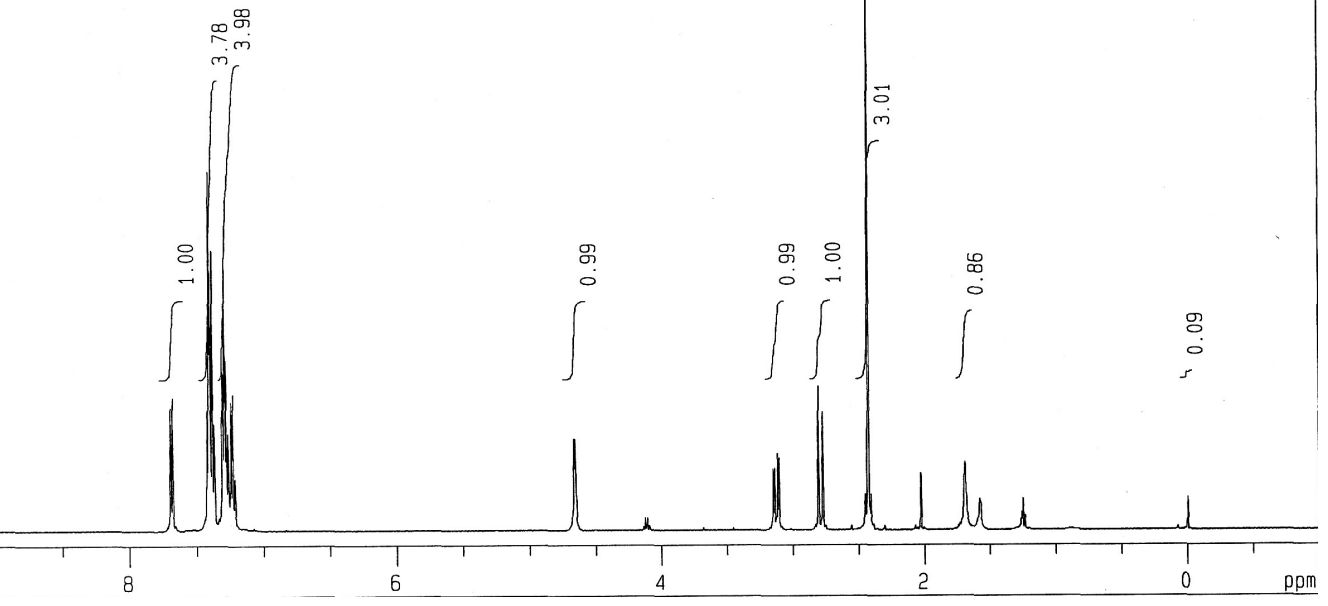



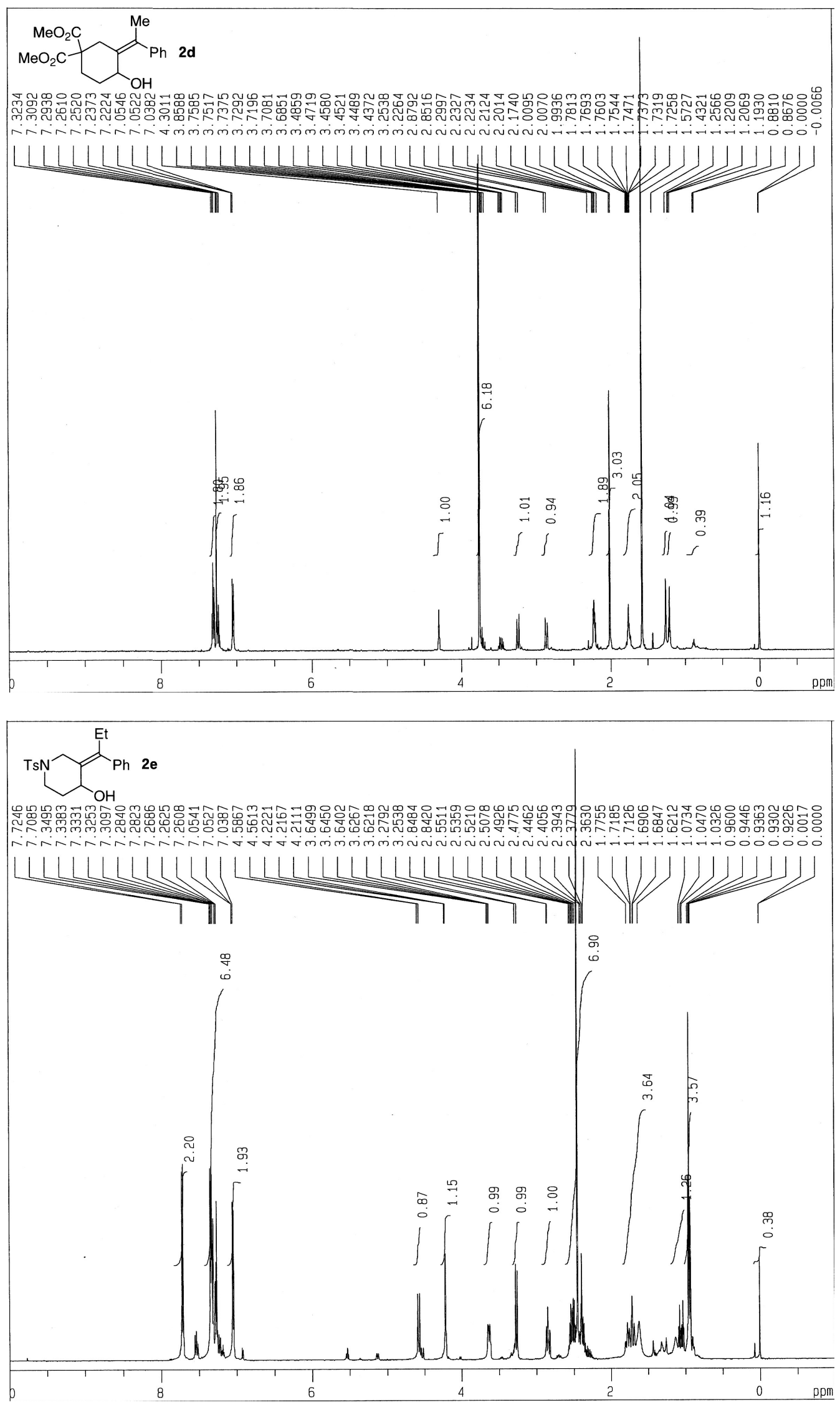

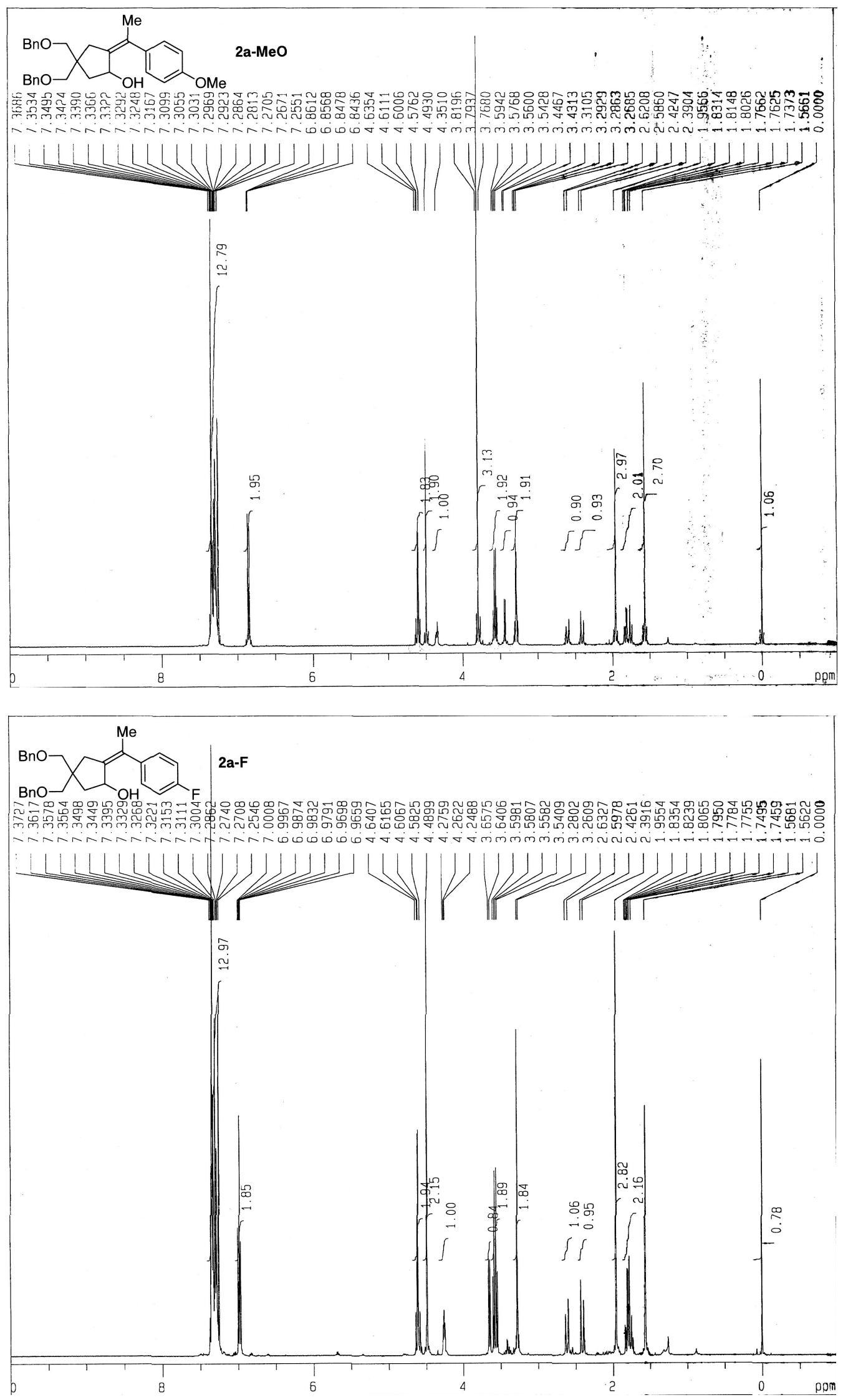

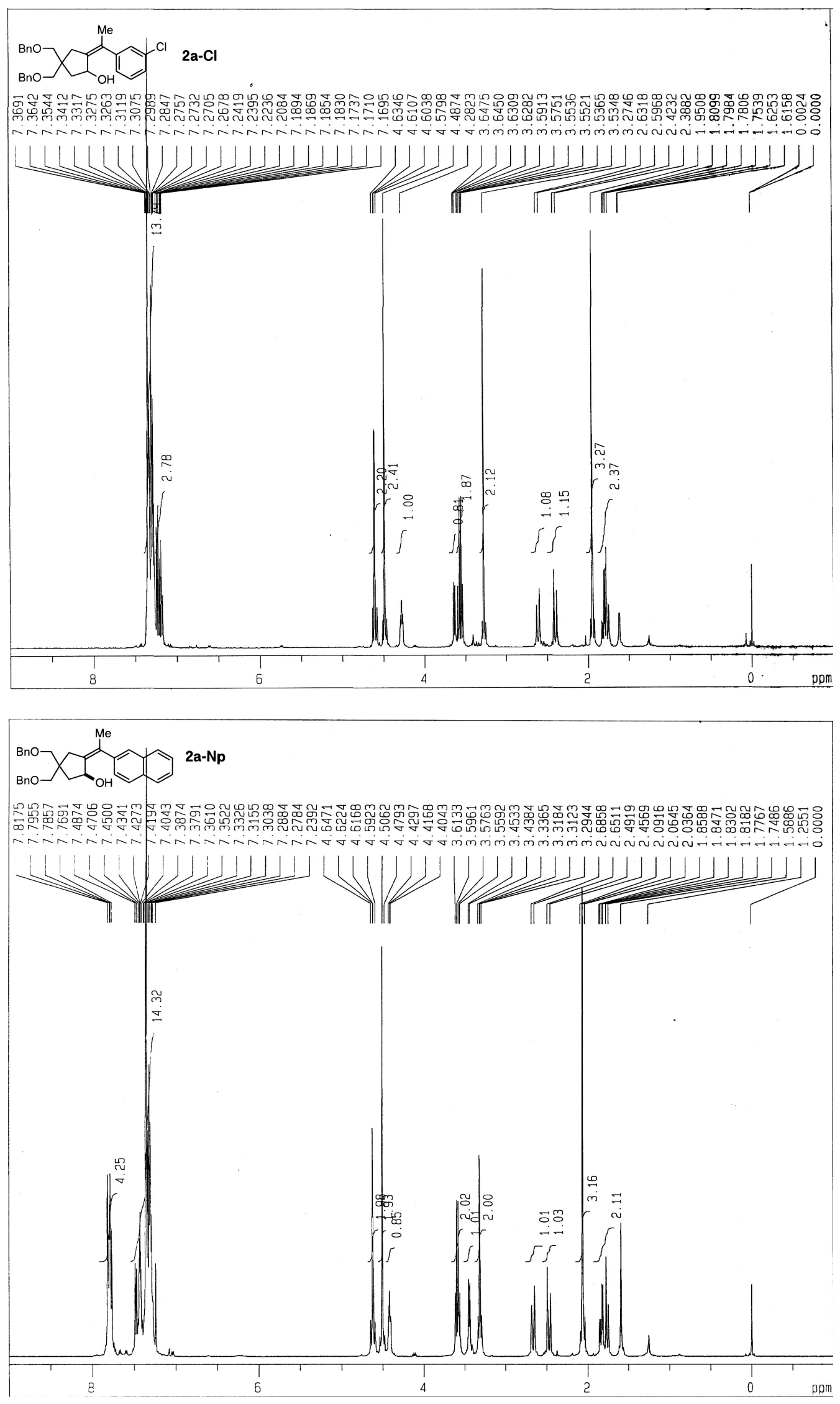

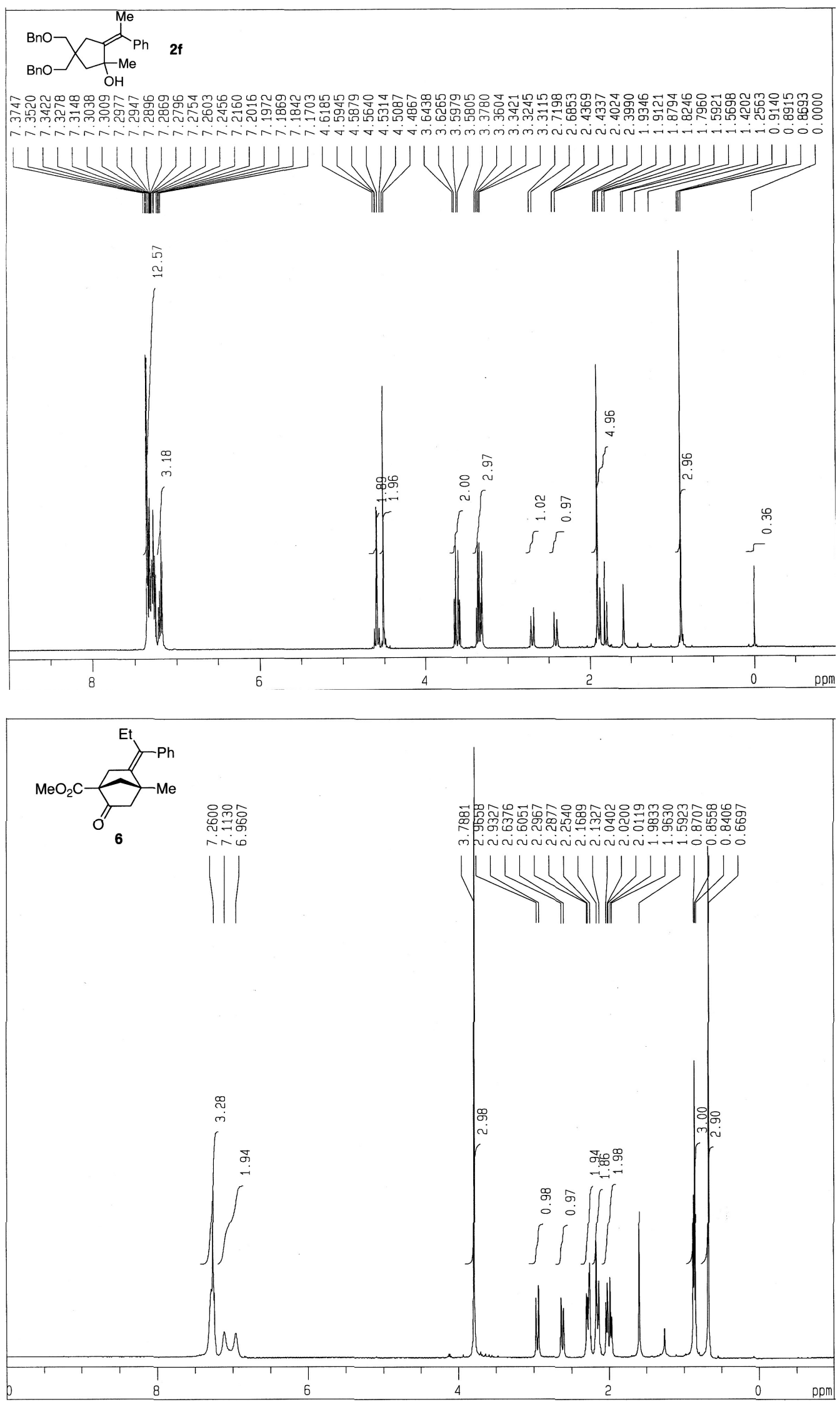